\title{
Far infrared properties of Hickson compact groups of galaxies. I. High resolution IRAS maps and fluxes ${ }^{\star}$
}

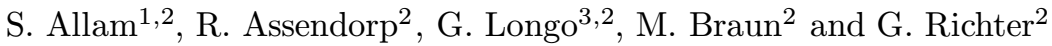 \\ 1 National Institut for Astronomy and Geophysics, Helwan, Egypt \\ 2 Potsdam Astrophysikalisches Institut, an der Sternwarte 16, Potsdam-Babelsberg, D-14482, Germany \\ 3 Osservatorio Astronomico di Capodimonte, via Moiariello 16, I-80131 Napoli, Italy
}

Received July 19; accepted October 27, 1995

\begin{abstract}
The Far Infrared (FIR) properties of galaxies which are members of compact groups bear relevant information on the dynamical status and the physical properties of these structures. All studies published so far have been undermined by the poor sensitivity and spatial resolution of the IRAS-PSC and IRAS Sky Survey data. We used the HIRAS software available at the IRAS server at the Laboratory for Space Research in Groningen to fully exploit the redundancy of the IRAS data and to approach the theoretical diffraction limit of IRAS. Among the 97 groups which were observed by IRAS, 62 were detected in at least one band, while reliable upper limits were derived for all the others. Among the detected groups, 49 were fully or partially resolved, i.e. it was possible to discriminate which member or members emit most of the FIR light. At $60 \mu \mathrm{m}$, for instance, 87 individual sources were detected in 62 groups. In order to ease the comparison with data obtained at other wavelengths - and in particular in the X and radio domains - we give co-added and HIRAS maps for all the detected groups.
\end{abstract}

Key words: atlases — galaxies: clusters of — infrared: galaxies

\section{Introduction}

With an average density of $10^{-24} \mathrm{gm} \mathrm{cm}^{-3}$, compact groups of galaxies listed in Hickson's (1982) catalogue are among the densest environments on extragalactic scale. Their average velocity dispersion of $150 \mathrm{~km} \mathrm{~s}^{-1}$ - which is less than one fifth of that observed in some cores of rich clusters of galaxies - renders the dynamical friction mechanism particularly effective and turns them into ideal test grounds where to study the effects of strong galactic interactions and, in some extreme cases, also of cannibalism and merging.

In spite of the large amount of observational and theoretical work done in the last decade, the physical properties of compact groups of galaxies (hereafter referred to as CGs) still present oblivious aspects (Trimble \& Leonard 1995) due to contradicting observational evidences. While referring to some recent reviews for up to date overviews of the field (Hickson 1993; Mamon 1995; Zepf 1995), we wish to focus here on the rather puzzling problems posed by the FIR luminosity function of the groups. Even though some debate on the details of the process still persists, it seems by now well established that during strong galaxy interactions, gas and dust may dissipate angular momentum and become more centrally concentrated thus triggering non-thermal nuclear activity and bursts of star formation

Send offprint requests to: $\mathrm{R}$. Assendorp

${ }^{\star}$ Table 2 is also available in electronic form at the CDS via anonymous ftp 130.79.128.5 which, in turn, affect both the colors (Larson \& Tinsley 1978) and the luminosities at radio (Stocke 1978), optical and infrared (Haynes \& Herter 1988; Bushhouse et al. 1988; Xu \& Sulentic 1991; Surace et al. 1993) wavelengths. The heating of the dust by the UV radiation emitted by the young, massive stars leads to an increase in the dust temperature (as measured by the $f_{60} / f_{100} \mu \mathrm{m}$ ratio) and to an excess of FIR luminosity (Telesco 1988; Londsdale et al. 1984). Surprisingly, while this FIR excess has been confirmed for interacting galaxies in pairs (Xu \& Sulentic 1991) and clusters (Bicay \& Giovannelli 1987), its existence in the dense environment of compact groups of galaxies is still dubious. An early study of CGs by Hickson and his collaborators (Hickson et al. 1989; hereafter HMPP) made use of the IRAS Point Source Catalogue (=IRAS-PSC) to find an enhancement comparable to that observed by Bicay \& Giovannelli (1987) in the cores of rich clusters of galaxies. Due to the rather high IRAS-PSC detection threshold and to the low spatial resolution, HMPP detected only the FIR brightest groups (i.e. $\sim 45 \%$ of the sample) and among these, only few were resolved into individual components, thus leaving often undecided which, among the members of the group, could be the true infrared sources. In these cases HMPP assumed the FIR emission to originate from one group member only. An assumption which was shown to lead to systematic errors by Sulentic \& De Mello Rabaca (1993) who, using the same data set as in HMPP and on the basis of statistical arguments only, reached the opposite conclusion that CG's 
galaxies could actually be normal or even under-luminous in the FIR.

In order to settle the argument on more stable observational grounds we have re-processed all IRAS data on Hickson Groups, fully exploiting the redundancy of the data and the accurate knowledge of the satellite Point Spread Function, reaching a much lower detection threshold, and approaching the diffraction limited angular resolution of the satellite.

In Sect. 2 we give some details on the data and the data reduction procedure and, in Sect. 3 we explain how fluxes and upper limits were derived. In Sect. 4 we describe the atlas. In Sect. 5 we give additional information on some of the groups in the sample and, finally in Sect. 6 we summarize the results. The astrophysical implication of the data will be discussed in a forthcoming paper (Assendorp et al. 1995c).

\section{Data reduction and analysis}

The IRAS (see the IRAS-Explanatory Supplement $=I R A S-E S$ for information about the mission) data where extracted from the server located at the Laboratory for Space Research in Groningen (Assendorp et al. 1995a). Most of the time, IRAS patrolled the sky running from one ecliptic pole to the other and, usually, a given position on the sky has been covered by more than one scan. In some cases also Pointed Observations (=POs; Assendorp $\&$ Wesselius 1993) were performed. Images in the IRAS Sky Survey have angular resolution of the order of $5^{\prime}$ in the cross-scan direction and of about $1.2^{\prime}$ in the in-scan direction, i.e. far higher than the theoretical resolution of the instrument which - as defined by the radius encircling $80 \%$ of the energy - is ca. 25, 25, 60 and $100^{\prime \prime}$ at 12,25 , 60 and $100 \mu \mathrm{m}$, respectively (IRAS-ES).

The HIRAS software available at the IRAS server node, is based on the Pyramid Maximum Entropy (=PME) method described by Bontekoe et al. (1994). PME makes use of the MEMSYS5 package (Gull \& Skilling 1991) and of the accurate knowledge of the IRAS point spread function. As a rule of thumb, for sources having in the coadded images a $S / N$ ratio better than 5 (the exact value depending on the structure of the background), HIRAS can be used to restore the angular resolution down to the theoretical diffraction limits of the instrument (see also Assendorp et al. 1995b).

The usefulness of this and similar methods (such as HIRES based on the Maximum Correlation Method, Aumann et al. 1990) has been discussed several times in the literature (cf. Surace et al. 1993; Rice 1993). We want to stress here that in the case of CGs the gain in sensitivity and in angular resolution are both crucial, due to the specific properties of the sample which is characterized by an average FIR luminosity close to the IRAS-PSC detection limit and by an average angular size of 3'6 i.e. smaller than the IRAS-PSC resolution.
Absolute positions provided by IRAS are accurate $( \pm 1 \sigma)$ within $2^{\prime \prime}$ in the in-scan direction and $\sim 8.5^{\prime \prime}$ in the crossscan direction.

No IRAS data were available for Groups 42,51 and 52 which happen to fall into that $5 \%$ of the sky which has not been observed by IRAS.

\section{Absolute flux calibration}

The absolute calibration of the IRAS data has been discussed many times in the literature and we refer to the IRAS-ES, to the introduction to the IRAS Faint Source Survey (=IRAS-FSS) and to Assendorp et al. (1995a), for a general overview of the procedure. We shall therefore stress only a few specific points.

\subsection{Non linearity}

The recently discovered non linearity in the IRAS calibration (Assendorp, private communication) becomes larger than the IRAS photometric accuracy (i.e. about 10\%) only at fairly high flux levels (of the order of $100 \mathrm{Jy}$ or more at $100 \mu \mathrm{m}$ ) and, therefore, it does not affect by any means the data discussed here, which are confined within the 10 Jy range (see also Fig. 1).

\subsubsection{Upper limits}

A source has been regarded as detected when its flux is above $3 \sigma$, where $\sigma$ is the rms noise of the background. This $3 \sigma$ value, once corrected for the beam factor, gives also the upper limit for undetected sources. In the case of MaxEnt images, due to the fact that the program alters the structure of the noise, $\sigma$ was computed from the original co-added images. In a few cases, i.e. when the inspection of the maps suggested the existence of a source very close to the detection treshold, the IRAS scans were investigated one by one to check whether the signal was produced by spurious noise (such as a particle event) into one of the scans or by a faint source. We followed the empirical rule that if the source was detected in more than one but less than half of the scans we took the upper limit, otherwise we took the average of the individual detections.

\subsubsection{IRAS-PSC or FSS objects}

When the group as a whole or some of its members had an accurate (i.e. error $<20 \%$ ) flux in either the IRASPSC or the IRAS-FSS, we integrated the flux over the corresponding source(s) in either the co-added or the MaxEnt images, and matched the derived flux to the catalogue value (when reliable entries were present in both the PSC and the FSS catalogue, the FSS data were preferred). 

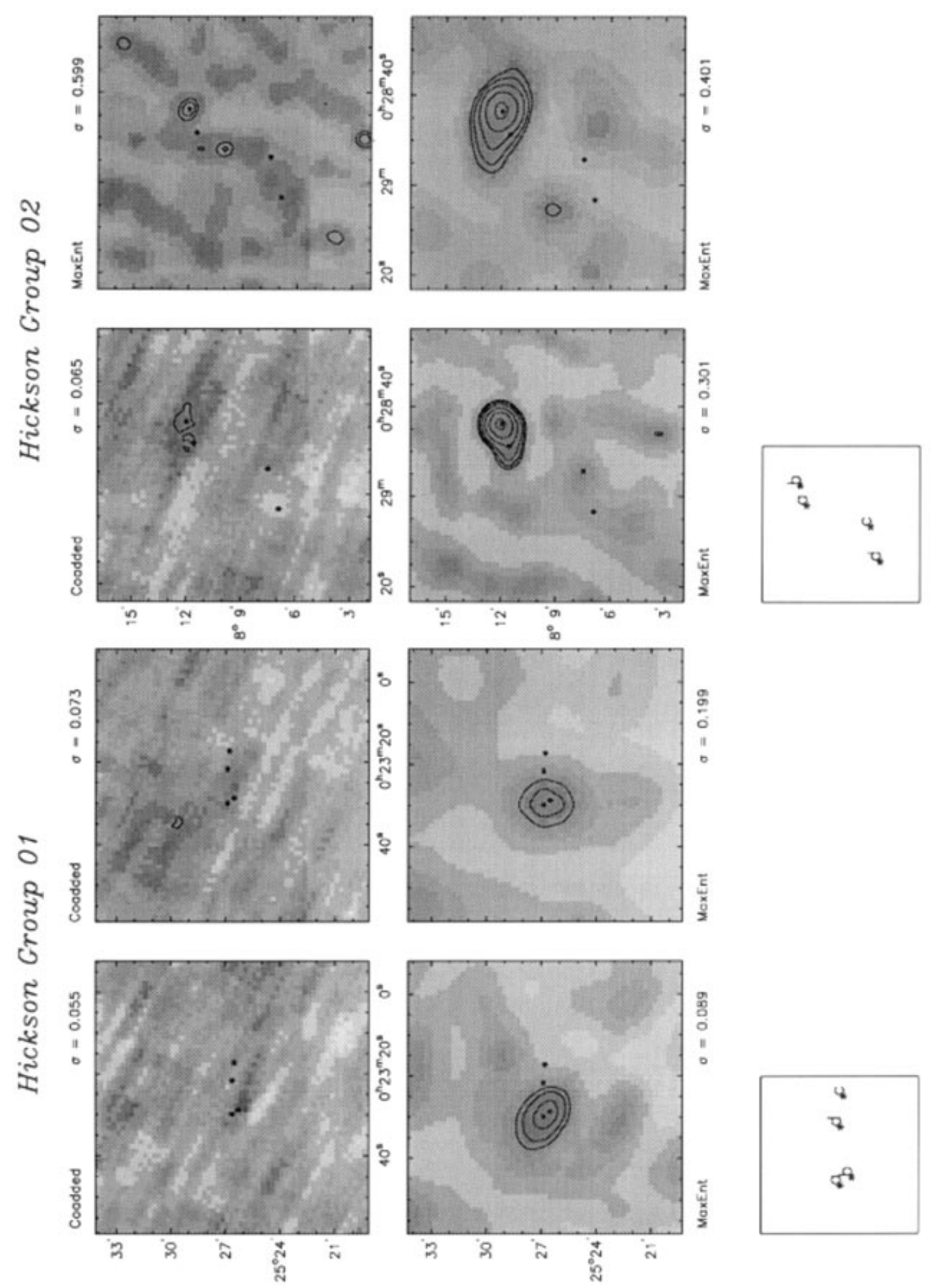

Fig. 1. Distribution (for each of the four IRAS bands) of the peak-pixel values ( $Y$-axis, MJy/sr) against the fluxes of the IRAS-PSC and FSS sources ( $X$-axis, Jy) present in our sample. In abscissa is the log of either the $f_{\mathrm{FSS}}$ or the $f_{\mathrm{PSC}}$ fluxes, while in ordinate is the log of the peak pixel value 


\subsubsection{Non IRAS-PSC or FSS sources}

If a group was detected in our images but no IRAS PSC or FSS sources were present in the field, we estimated the fluxes from the co-added IRAS scans.

\subsubsection{Extended sources}

In some cases (depending on the relative luminosities and on the angular separation) sources were partially but not fully resolved by Max-Ent. In these cases we obtained a profile along the line joining the two maxima and fitted two or more Gaussians to the profile in order to separate the contribution from the two components. Individual fluxes were then obtained by parting the total flux accordingly to the peak-pixels ratios. In Fig. 1 we show for each of the four IRAS bands the correlation between peak-pixel value and the PSC or FSS fluxes. As it can be seen, the correlations are linear over the whole range and the scatter of the points is rather small. Fluxes are listed in Table 2, which is structured as follows:

- Column 1: identification according to Hickson (1982). If the group is not detected in any of the IRAS bands there is only one entry.

- Column 2: Morphological type of the galaxy according to Hickson (1993).

- Columns 3-6: fluxes and related errors in the 12, 25, 60 and $100 \mu \mathrm{m}$ bands, respectively. Fluxes are in Jy. When the FIR source corresponds to several unresolved members of the group we give (in brackets) the list of the members which fall within the FIR source. This value is given for only one of the objects forming the unresolved structure. Errors are given according to codes similar to those used in the IRAS-PSC: 3 denotes errors of the same order of the IRAS photometric accuracy $(\sim 10 \%) ; 2$ errors within $20 \%$ and 1 for larger errors.

- Column 7: Notes to the FIR maps. POs available meaning that POs have been taken into account and processed together with the co-added scans.

\section{The Atlas}

In the Atlas we give the maps of those groups which have been detected in at least one of the four IRAS bands. For those cases where the group galaxies are not detected we show the co-added images, otherwise we show the MaxEnt images. Due to the large spread in the angular sizes of the groups it was not possible to render all maps on the same scale. In order to easy the comparison between maps of different groups, we used only two scales (in a ratio 1:2). Axes correspond to the R.A. and Dec (Epoch=1950.0).

Grey and contours levels are given in steps of noise ( $\sigma$ in units $\mathrm{MJy} / \mathrm{sr}$ ) above the background: the first six levels $(-3,-2,-10,1,2$ in units of $\sigma)$ are mainly dominated by the large scale fluctuations of the background and are plotted as grey levels; while structures $3 \sigma$ and above are plotted as iso-contours (corresponding to 4, 6, 10, 20, 40, 80 in units of $\sigma$ ). The value of $\sigma$ and the type of map (i.e. either co-added or Max-Ent) are noted on two opposite sides of the maps. In all plots, crosses mark the optical positions of the various group members as listed in Hickson (1993). The lower left box allows the identification of the various group members according to the codes introduced by Hickson (1982). Maps are ordered as follows: $12 \mu \mathrm{m}$ (upper left corner); $25 \mu \mathrm{m}$ (upper right); 60 $\mu \mathrm{m}$ (lower left); $100 \mu \mathrm{m}$ (lower right). The fact that most $25 \mu \mathrm{m}$ maps are noisier than those at other wavelengths is related to the lower performance of the corresponding IRAS detectors.

\section{Comments on individual group}

Over the last decade a large database has cumulated on the overall properties of CGs, allowing us to discriminate between optical and physical groups and providing us with useful information on their emission properties in various wavelength ranges. In this section we give additional information - such as group memberships, radio detections etc.. - which are needed to better understand the maps and which will be used in Paper II. Unless otherwise explicitly noted, the non FIR data are taken from Hickson (1993).

Hickson 1 - Interacting pair $a b$; the group might be part of a loose group; $a b$ detected, but not resolved.

Hickson 2 - Object $d$ is at much higher redshift and is not detected in the FIR. The remaining triplet is accordant and has a disturbed optical morphology. Object $b$ is the stronger FIR source, but its elongated structure at $60 \mu \mathrm{m}$ suggests that the contribution from $a$ cannot be neglected at longer wavelengths.

Hickson 3 - Loose triplet $(a, b$ and $d) ; c$ is at much higher redshift and is also the brightest FIR source. At $100 \mu \mathrm{m}$ object $a c$ is not fully resolved, but the source is clearly extended and independent flux estimates could be derived.

Hickson 4 - Accordant triplet $(a, c$ and $d)$. In spite of the rather large redshift difference, object $b$ might also be a member. Object $e$ has a far too high redshift. At FIR wavelengths $a$ is clearly detected in all bands; $d$ is marginally detected at $60 \mu \mathrm{m}$.

Hickson 7 - Group of four bright (all are NGC objects) interacting galaxies. Objects $a$ and $c$ are strong FIR sources.

Hickson 9 - Galaxies do not form a physically bound group; object $a$ is a radio source. Our data suggest that FIR emission comes from object $d$. The elongation of the isophotes observed at $100 \mu \mathrm{m}$ is an artifact introduced by the background.

Hickson 10 - Rather loose group, but $a, b$ and $c$ seem to be interacting. Both $a$ and $c$ are detected at 60 and $100 \mu \mathrm{m}$.

Hickson 11 - Group is a chance alignment of unrelated objects and the FIR source $a$ is a fairly isolated object. 
Hickson 14 - Galaxy $d$ has higher redshift than the other members; $a$ is a strong FIR source; HMPP attribute the FIR emission to $b$ which is not detected in our maps. The source at $12 \mu \mathrm{m}$ is a star.

Hickson 16 - Also known as Arp 318. All objects but $b$ are strong radio and IR sources.

Hickson 18 - Also known as Arp 258 or VV 143. Object $a$ has higher redshift. Object $b c d$ detected but unresolved at 60 and $100 \mu \mathrm{m}$.

Hickson 19 - Group is detected at 60 and $100 \mu \mathrm{m} ; b$ is the brightest FIR source.

Hickson 21 - Loose triplet $a, b$ and $c$, plus a pair at higher redshift. $a$ is a bright FIR source; $b$ is only marginally detected at 60 and $100 \mu \mathrm{m}$. The $25 \mu \mathrm{m}$ map is very noisy.

Hickson 23 - Object $e$ is at much higher redshift. According to HMPP galaxies $b$ and $d$ are the FIR sources. $b$ is a radio source.

Hickson 25 - Accordant quartet $(a, b, d$ and $f)$ plus higher redshift triplet; $a$ and $c e$ are IR sources, $a$ is also a radio source.

Hickson 26 - Dense group. Galaxy $a$ is a radio source; $a b d g$ form an unresolved bright FIR source.

Hickson 27 - Quartet of accordant high redshift objects $(c, d, e, f)$ plus a lower redshift pair. The quartet is unresolved but detected at the two longer wavelenghts. Also object $b$ is detected.

Hickson 29 - High redshift group; clear detection at 60 $\mu \mathrm{m}$; marginally detected at $100 \mu \mathrm{m}$.

Hickson 31 - Group of strongly interacting galaxies. $d$ is at much higher redshift. Object $c$ (Mrk 1089) is a peculiar object with emission lines and a central near IR bright source. The group is detected but unresolved at 60 and $100 \mu \mathrm{m}$. At $25 \mu \mathrm{m}$ only $c a$ is detected.

Hickson 33 - Accordant quartet. $a$ is a relatively strong radio source, $c$ is a weak radio source. The pair $a c$ is marginally detected at 60 and $100 \mu \mathrm{m}$.

Hickson 34 - Known as Arp 327 or VV169. Galaxies $a$, $b$ and $c$ are radio sources.

Hickson 36 - All objects have rather different redshifts. Chance alignment of physically unrelated objects. In any case, the group is detected but unresolved.

Hickson 37 - Objects $a$ and $b$ are radio sources; $b$ is a near IR source. In our maps the group $b c d$ is unresolved but extended

Hickson 38 - Contains the interacting pair ( $b$ and $c$ ) known as Arp 237; $d$ has much higher redshift. Both the pair $b c$ and $a$ are detected at $60 \mu \mathrm{m}$.

Hickson 39 - Small chain of high redshift objects detected only at $60 \mu \mathrm{m}$.

Hickson 40 - Very compact quintet of radio galaxies. Objects $a, c$ and $d$ are radio sources. HMPP identify $e$ with the FIR source. In our HIRAS maps the group bce is detected but unresolved. The $25 \mu \mathrm{m}$ map is rather noisy.

Hickson 41 - All galaxies have quite different redshift and thus are likely physically unrelated. $a$ is the dominant FIR source. At $60 \mu$ also the contribution from $b$ could be separated.

Hickson 43 - Quintet of mostly late type objects; $f$ is at much higher redshift; $a$ and $b$ are bright FIR sources.

Hickson 44 - Objects $a, c$ and $d$ are radio and FIR sources; All maps are noisy.

Hickson 45 - Rather dispersed compact group which might belong to a loose group; ad detected but unresolved.

Hickson 47 - Accordant quartet. Both $a$ and $b$ are radio sources; $b$ is rather compact while $a$ has a ring of radio emission. FIR emission originates from these two unresolved objects.

Hickson 48 - Object $b$ detected at 60 and $100 \mu \mathrm{m}$. The $12 \mu \mathrm{m}$ is noisy.

Hickson 49 - Small and very compact quartet marginally detected at the longer wavewlengths.

Hickson 53 - Galaxy $d$ has higher redshift; $a$ is a radio source; $a$ and $b c$ detected at $60 \mu \mathrm{m}$. A bright source North of $a$ remains unidentified. $a$ is detected also at $100 \mu \mathrm{m}$.

Hickson 54 - Group detected but unresolved at 60 and $100 \mu \mathrm{m}$.

Hickson 55 - Compact chain of mostly early-type objects, detected but unresolved at 60 and $100 \mu \mathrm{m}$.

Hickson 56 - Objects $b$ and $d$ are radio sources. $b c d$ detected at 25, 60 and $100 \mu \mathrm{m}$.

Hickson $\mathbf{5 7}$ - Populous group of 8 galaxies (Arp 320, VV 282). The compact galaxy $d$ is a radio source; $d a$ is detected but unresolved at 60 and $100 \mu \mathrm{m}$.

Hickson 58 - Rather loose group; $a$ and $c$ are radio sources; $b$ and $a$ are detected at 60 and $100 \mu \mathrm{m}$. The $25 \mu \mathrm{m}$ map is noisy.

Hickson 59 - Accordant quartet plus fainter and higher redshift galaxy (object $e$ ); $a$ is a radio source; $d$ is a rather strong FIR source. The $25 \mu \mathrm{m}$ map is noisy.

Hickson $\mathbf{6 1}$ - The group is actually a triplet (galaxy $b$ is foreground). Galaxy $c$ is both a FIR and a radio source; At $100 \mu \mathrm{m}$ also $a$ might be detected.

Hickson 63 - Relatively loose triplet $(b, c$ and $d)$ plus a low redhift object; $a$ and $d$ detected at $60 \mu \mathrm{m}$.

Hickson $67-$ Galaxy $b$ is detected at 60 and $100 \mu \mathrm{m}$. It has also some radio emission.

Hickson 68 - Very bright star South of galaxy $c$ affects the $12 \mu \mathrm{m}$ map. Object $c$ and the pair $a b$ are detected.

Hickson 69 - Accordant and very compact quartet (VV 281). It is likely part of a loose group. Objects $a$ and $b$ are radio sources; $b$ is detected at 60 and $100 \mu \mathrm{m}$. 
Hickson 70 - Group is actually formed by an accordant triplet of galaxies $(a, b$ and $c)$ plus an accordant quartet at much higher redshift. At $60 \mu$ object $b$ is the brightest FIR source, at $100 \mu \mathrm{m}$ ad are brighter.

Hickson 71 - Galaxies $a, b$ and $c$ form an accordant triplet. Object $d$ has much (factor 3 ) higher redshift. Object $b$ is detected at 60 and $100 \mu \mathrm{m}$.

Hickson 73 - Group is actually formed by a bright low redshift galaxy - detected also at radio wavelengths - plus an accordant triplet at much higher redshift. Galaxy $e$ is a background object. FIR emission likely originates from galaxy $a$ only.

Hickson 75 - cde detected at 60 and $100 \mu \mathrm{m}$.

Hickson $\mathbf{7 7}$ - The group is formed by an high redshift pair $a b$, plus a second, lower redshift interacting pair $c d$; $c d$ are marginally detected at $60 \mu \mathrm{m}$.

Hickson 78 - Not a group. Galaxies $a$ and $b$ may form an interacting pair (distortions are clearly visible); $c$ might be detected at $60 \mu \mathrm{m}$.

Hickson 79 - Known also as Seyfert Sextet (VV 115), it is also the most compact among Hickson's list. Hickson (1993b) attributes FIR emission to galaxy $a$ only (it is also a radio source). Unresolved.

Hickson 80 - Accordant quartet of three late type (a, $c$, and $d$ ) plus an early type object; unresolved by IRAS beam. Galaxy $a$ is a radio source. $a$ and $b$ are detected but unresolved at 25, 60 and $100 \mu \mathrm{m}$.

Hickson 81 - Compact quartet at high redshift; $a$ is a radio source. Group marginally detected at $60 \mu \mathrm{m}$.

Hickson 82 - Rather loose group. $c$ is also a radio source; $c d$ detected at 60 and $100 \mu \mathrm{m}$.

Hickson 88 - Relatively loose quartet; $a$ and $d$ are radio sources; $a$ and $c$ are detected at 60 and $100 \mu \mathrm{m}$.

Hickson 89 - Possible detection of galaxies $c$ and $d$ at $60 \mu \mathrm{m}$.

Hickson 90 - Strongly interacting system. Galaxy $a$ is well resolved in all four IRAS bands. A second FIR source coincides with galaxies $b, c$ and $d$. Since detailed multiband CCD photometry shows no signs of cold dust in galaxies $b$ and $c$, the FIR emission very likely originates from galaxy $d$ (i.e. NGC 7174: a late type spiral heavily disrupted by the ongoing interaction with galaxy $c$, namely NGC 7173) only.

Hickson $91-a$ and $d$ form an interacting pair, which is detected at 60 and $100 \mu \mathrm{m}$.

Hickson 92 - Better known as Stephan's Quintet (Arp 319, VV 228). $a$ and $c$ are resolved at $60 \mu \mathrm{m}, c$ is the only source detected at $100 \mu \mathrm{m}$. The $25 \mu \mathrm{m}$ map is rather noisy.

Hickson 93 - Galaxy $b$ is detected in all bands.

Hickson 95 - Unresolved in our maps. In HMPP, galaxy $d$ is said to be the IR source.

Hickson 96 - ac pair detected in all four bands.
Hickson 100 - Redshift of galaxy $d$ is missing. Morphology however, makes it very likely a background object. $b a$ are detected as an extended but not resolved source.

Table 1. Comparison of the number of groups and of individual sources detected in the IRAS-PSC, the IRAS-FSS and in the present work, respectively. For each entry we give for each band (Cols. 2-5), on the first line the number of groups detected and, in brackets, the number of individual sources. On the second line, we give the average detection treshold in $\mathrm{Jy}$

\begin{tabular}{l|cccc|}
\hline \hline Sample & $12 \mu \mathrm{m}$ & $25 \mu \mathrm{m}$ & $60 \mu \mathrm{m}$ & $100 \mu \mathrm{m}$ \\
& & & & \\
\hline \hline IRAS-PSC & $4(4)$ & $7(8)$ & $34(40)$ & $43(49)$ \\
& 0.4 & 0.5 & 0.6 & 1.0 \\
\hline IRAS-FSS & $14(17)$ & $11(12)$ & $58(66)$ & - \\
& 0.1 & 0.09 & 0.225 & 1.0 \\
\hline this work & $17(20)$ & $24(27)$ & $62(87)$ & $57(74)$ \\
& 0.048 & 0.073 & 0.159 & 0.620 \\
\hline \hline
\end{tabular}

\section{Summary}

We have used the software available at the Laboratory for Space Research of Groningen to obtain high resolution, high sensitivity maps of all compact groups contained in the Hickson list (Hickson 1982). The higher sensitivity of our survey (see Table 1 for a comparison of our survey with respect to the IRAS-PSC and IRAS -FSS) allowed us to detect 62 out of the 97 groups observed by IRAS. For most of these groups we were able to discriminate at least in part which members the FIR radiation is coming from. A detailed discussion of the FIR luminosities, luminosity functions, and colors, as well as of the correlations of the FIR parameters with other observable quantities will be given in Assendorp et al. (1995).

Acknowledgements. The IRAS data were obtained using the server at the Space Research Laboratory, Groningen, The Netherlands, and the Dutch expertise Center for Astronomical Data Processing funded by the Nederlands Organization for Scientific Research (NWO). The IRAS data base server project was also partly funded through the Air Force Office of Scientific Research (grants AFOSR 86-0140 and 89-0320). The processing has been carried out making use of the GIPSY (Groningen Image Processing SYstem) software. GL acknowledges the Astrophysikalisches Institut Potsdam, the Italian Space Agency (ASI) and the Italian CNR for financial support. SA was supported by the DAAD (Deutscher Akademischer Austauschdienst) grant No. 562 .

\section{References}

Assendorp R., Bontekoe T.R., de Jonge A.R.W., et al., 1995a, A\&A 110, 395

Assendorp R., Longo G., Kester D., Richter G., 1995b, A\&A (in preparation)

Assendorp R., Longo G., Allam S., 1995c (in preparation) 
Assendorp R., Wesselius P.R., 1993, A\&AS 100, 473

Aumann H.H., Fowler J.W., Melnyk M., 1990, AJ 99, 1674

Bicay X, Giovannelli R., 1987, ApJ 321, 645

Bontekoe T.R., Koper E., Kester D.J.M., 1994, A\&A 284, 1037

Bushhouse et al. 1988

Gull S.F., Skilling J., 1991, MEMSYS5 User's Manual

Haynes M.P., Herter T., 1988, AJ 96, 504

Hickson P., 1982, ApJ 255, 382

Hickson P., 1993, Atlas of Compact Groups of Galaxies, Astrophys. Lett. Commun. 29, 1

Hickson P., Menon T.K., Palumbo G.C.G., Persic M., 1989, ApJ 341, 679 (=HMPP)

IRAS Catalogues and Atlases: Vol. 1, Explanatory Supplement, 1988, in: Beichman C.A., Neugebauer G., Habing H.J., Clegg P.E., Chester T.J. (eds.), NASA RP-1190, Washington DC (=IRAS-ES)

IRAS Faint Source Survey, Version 2, Explanatory Supplement, 1992, in: Moshir M., Kopan G., Conrow T.,
McCallon H., Hacking P., Gregorich D., Rohrbach G., Melnyk M., Rice W., Fullmer L., White J., Chester T. (eds.), JPL Pasadena (=IRAS-FSS)

IRAS Point Sources Catalogue, Version 2, 1988, Joint IRAS Science Working Group (eds.) GPO, Washington DC (=IRAS-PSC)

Larson R.B., Tinsley B.M., 1978, ApJ 219, 46

Londsdale C., Persson E., Matthews K., 1984, ApJ 287, 95

Mamon G.A., 1995, in Groups of Galaxies, Astron. Soc. of Pac. Conf. Ser. No. 70 , p. 83

Rice W., 1993, AJ 105, 67

Stocke J.T., 1978, AJ 83, 348

Sulentic J.W., De Mello Rabaca D., 1993, ApJ 410, 520 $(=\mathrm{SDM})$

Sulentic J.W., Rabaca C.R., 1994, ApJ 429, 531

Sulentic J.W., Rabaca C.R., 1995, Astron. Soc. of Pac. Conf. Ser. No. 70, p. 127

Surace J.A., Mazzarella J., Soifer B.T., Wehrle A.E., 1993, AJ 105,864

Telesco C., Wolstencroft R., Done C., 1988, ApJ 329, 174

Trimble V., Leonard P.J.T., 1995, PASP 107, 1

Xu C., Sulentic J., 1991, ApJ 374, 407

Zepf S.E., 1995, in Groups of Galaxies, Astron. Soc. of Pac. Conf. Ser. No. 70, p. 135 
Table 2. Fluxes and upper limits for galaxies which are member of the Hickson Groups

\begin{tabular}{|c|c|c|c|c|c|c|c|c|}
\hline Ident. & $\mathrm{T}$ & $12 \mu \mathrm{m}$ & & $25 \mu \mathrm{m}$ & $60 \mu \mathrm{m}$ & & $100 \mu \mathrm{m}$ & Comments \\
\hline $1 \mathrm{a}$ & $\mathrm{Sc}$ & $<.05$ & & $<.05$ & $0.34(\mathrm{ab})$ & 3 & $0.94(\mathrm{ab}) 2$ & P.O.'s available. \\
\hline $1 \mathrm{~b}$ & $\mathrm{Im}$ & $<.05$ & & $<.05$ & & & & \\
\hline $1 \mathrm{c}$ & E0 & $<.05$ & & $<.05$ & $<.11$ & & $<.40$ & \\
\hline $1 d$ & S0 & $<.05$ & & $<.05$ & $<.11$ & & $<.40$ & \\
\hline $2 \mathrm{a}$ & SBd & $<.05$ & & $<.11$ & $\begin{array}{ll}1.26 & 3\end{array}$ & & $\begin{array}{ll}0.89 & 2\end{array}$ & \\
\hline $2 \mathrm{~b}$ & $\mathrm{cI}$ & 0.07 & 2 & $0.40 \quad 3$ & $2.34 \quad 3$ & & $4.49 \quad 2$ & \\
\hline $2 \mathrm{c}$ & $\mathrm{SBc}$ & $<.05$ & & $<.11$ & $<.14$ & & $<.78$ & \\
\hline $2 \mathrm{~d}$ & $\mathrm{SBb}$ & $<.05$ & & $<.11$ & $<.14$ & & $<.78$ & \\
\hline $3 a$ & $\mathrm{Sc}$ & $<.06$ & & $<.11$ & $0.20 \quad 3$ & & $0.39 \quad 2$ & \\
\hline $3 \mathrm{~b}$ & SB0a & $<.06$ & & $<.11$ & $<.05$ & & $<.37$ & \\
\hline $3 \mathrm{c}$ & $\mathrm{Sd}$ & $<.06$ & & $<.11$ & $0.53 \quad 3$ & & $0.65 \quad 2$ & \\
\hline $3 \mathrm{~d}$ & S0 & $<.06$ & & $<.11$ & $<.11$ & & $<.37$ & \\
\hline $4 a$ & Sc & 0.22 & 3 & $0.56 \quad 3$ & $3.85 \quad 3$ & & $8.08 \quad 3$ & \\
\hline $4 \mathrm{~b}$ & $\mathrm{Sc}$ & $<.08$ & & $<.07$ & $<.10$ & & $<.24$ & \\
\hline $4 \mathrm{c}$ & E2 & $<.08$ & & $<.07$ & $<.10$ & & $<.24$ & \\
\hline $4 d$ & $\mathrm{E} 4$ & $<.08$ & & $<.07$ & $0.21 \quad 2$ & & $<.24$ & \\
\hline $4 \mathrm{e}$ & $\mathrm{Sab}$ & $<.08$ & & $<.07$ & $<.10$ & & $<.24$ & \\
\hline 5 & & $<.06$ & & $<.06$ & $<.30$ & & $<.53$ & Not detected. \\
\hline 6 & & $<.05$ & & $<.11$ & $<.13$ & & $<.46$ & Not detected. \\
\hline $7 \mathrm{a}$ & $\mathrm{Sb}$ & 0.17 & 2 & $0.44 \quad 2$ & 3.093 & & $\begin{array}{ll}6.21 & 3\end{array}$ & \\
\hline $7 \mathrm{~b}$ & SB0 & $<.08$ & & $<.16$ & $<.37$ & & $<.43$ & \\
\hline $7 \mathrm{c}$ & $\mathrm{SBc}$ & $<.08$ & & $<.16$ & $0.60 \quad 3$ & & $2.13 \quad 2$ & \\
\hline $7 \mathrm{~d}$ & $\mathrm{SBc}$ & $<.08$ & & $<.16$ & $<.37$ & & $<.43$ & \\
\hline 8 & & $<.05$ & & $<.11$ & $<.13$ & & $<.42$ & Not detected. \\
\hline $9 \mathrm{a}$ & E2 & $<.05$ & & $<.07$ & $<.11$ & & 1.32 (abcd) 3 & \\
\hline $9 \mathrm{~b}$ & S0 & $<.05$ & & $<.07$ & $<.11$ & & & \\
\hline $9 \mathrm{c}$ & $\mathrm{Sc}$ & $<.05$ & & $<.07$ & $<.11$ & & & \\
\hline $9 \mathrm{~d}$ & SBcd & $<.05$ & & $<.07$ & $0.44 \quad 2$ & & & \\
\hline $10 \mathrm{a}$ & $\mathrm{SBb}$ & $<.04$ & & $0.09 \quad 2$ & $0.23 \quad 2$ & & $<.80$ & \\
\hline $10 \mathrm{~b}$ & E1 & $<.04$ & & $<.05$ & $<.16$ & & $<.80$ & \\
\hline $10 \mathrm{c}$ & $\mathrm{Sc}$ & $<.04$ & & $<.05$ & $0.76 \quad 3$ & & $2.00 \quad 3$ & \\
\hline $10 \mathrm{~d}$ & Scd & $<.04$ & & $<.05$ & $<.16$ & & $<.80$ & \\
\hline $11 \mathrm{a}$ & SBbc & $<.05$ & & $<.07$ & $\begin{array}{ll}0.31 & 3\end{array}$ & & $\begin{array}{ll}1.29 & 3\end{array}$ & \\
\hline $11 b$ & $\mathrm{SBc}$ & $<.05$ & & $<.07$ & $<.11$ & & $<.34$ & \\
\hline $11 \mathrm{c}$ & Scd & $<.05$ & & $<.07$ & $<.11$ & & $<.34$ & \\
\hline $11 d$ & SB0 & $<.05$ & & $<.07$ & $<.11$ & & $<.34$ & \\
\hline 12 & & $<.06$ & & $<.10$ & $<.15$ & & $<.80$ & Not detected. \\
\hline 13 & & $<.06$ & & $<.10$ & $<.11$ & & $<.53$ & Not detected. \\
\hline $14 \mathrm{a}$ & $\mathrm{Sb}$ & $<.05$ & & $<.10$ & $0.80 \quad 3$ & & $1.87 \quad 3$ & \\
\hline $14 b$ & E5 & $<.05$ & & $<.10$ & $<.11$ & & $<.34$ & \\
\hline $14 \mathrm{c}$ & $\mathrm{Sbc}$ & 0.28 & 3 & $<.10$ & $<.11$ & & $<.34$ & \\
\hline $14 \mathrm{~d}$ & $\mathrm{Sd}$ & $<.05$ & & $<.10$ & $<.11$ & & $<.34$ & \\
\hline 15 & & $<.06$ & & $<.11$ & $<.13$ & & $<.38$ & P.O.s available. \\
\hline $16 \mathrm{a}$ & SBab & 0.35 & 2 & $0.45 \quad 2$ & 5.313 & & $17.68 \quad 3$ & \\
\hline $16 \mathrm{~b}$ & $\mathrm{Sab}$ & $<.06$ & & $<.10$ & $<.12$ & & $<.33$ & \\
\hline $16 \mathrm{c}$ & $\mathrm{Im}$ & 0.54 & 3 & 1.843 & $9.23 \quad 3$ & & $20.79 \quad 3$ & \\
\hline $16 \mathrm{~d}$ & $\mathrm{Im}$ & 0.45 & 3 & $2.48 \quad 3$ & $10.55 \quad 3$ & & $23.57 \quad 3$ & \\
\hline 17 & & $<.05$ & & $<.08$ & $<.30$ & & $<1.98$ & Not detected. \\
\hline $18 \mathrm{a}$ & S0a & $<.05$ & & $<.24$ & $<.11$ & & $\begin{array}{ll}0.24 & 2\end{array}$ & \\
\hline $18 \mathrm{~b}$ & $\operatorname{Im}$ & $<.05$ & & $<.24$ & $0.51(\mathrm{bcd})$ & 2 & $0.80(\mathrm{bcd}) \quad 2$ & \\
\hline $18 \mathrm{c}$ & $\mathrm{Im}$ & $<.05$ & & $<.24$ & & & & \\
\hline $18 \mathrm{~d}$ & $\mathrm{Im}$ & $<.05$ & & $<.24$ & & & & \\
\hline
\end{tabular}


Table 2. continued

\begin{tabular}{|c|c|c|c|c|c|c|}
\hline Ident. & $\mathrm{T}$ & $12 \mu \mathrm{m}$ & $25 \mu \mathrm{m}$ & $60 \mu \mathrm{m}$ & $100 \mu \mathrm{m}$ & Comments \\
\hline $19 \mathrm{a}$ & $\overline{\mathrm{E} 2}$ & $<<.04$ & $<<.06$ & $<<.09$ & $<.38$ & \\
\hline $19 b$ & Scd & $<.04$ & $<.06$ & 0.30 & 1.11 & \\
\hline $19 \mathrm{c}$ & $\mathrm{Sdm}$ & $<.04$ & $<.06$ & $<.09$ & $<.38$ & \\
\hline $19 \mathrm{~d}$ & $\mathrm{SBb}$ & $<.04$ & $<.06$ & $<.09$ & $<.38$ & \\
\hline 20 & & $<.06$ & $<.08$ & $<.23$ & $<1.43$ & Not detected. \\
\hline $21 \mathrm{a}$ & $\mathrm{Sc}$ & $0.11 \quad 2$ & $0.19 \quad 2$ & $1.06 \quad 3$ & $3.13 \quad 3$ & \\
\hline $21 b$ & $\mathrm{Sab}$ & $<.08$ & $<.05$ & $0.12 \quad 2$ & $0.83 \quad 3$ & \\
\hline $21 \mathrm{c}$ & E1 & $<.08$ & $<.05$ & $<.10$ & $<.46$ & \\
\hline $21 d$ & $\mathrm{E} 2$ & $<.08$ & $<.05$ & $<.10$ & $<.46$ & \\
\hline $21 \mathrm{e}$ & SB0a & $<.08$ & $<.05$ & $<.10$ & $<.46$ & \\
\hline 22 & & $<.04$ & $<.05$ & $<.11$ & $<1.48$ & Not detected. \\
\hline $23 a$ & Sab & $<.04$ & $<.06$ & $<.14$ & $<.95$ & \\
\hline $23 \mathrm{~b}$ & $\mathrm{SBc}$ & $<.04$ & $<.06$ & 1.392 & $3.94 \quad 3$ & \\
\hline $23 \mathrm{c}$ & S0 & $<.04$ & $<.06$ & $<.14$ & $<.95$ & \\
\hline $23 \mathrm{~d}$ & $\mathrm{Sd}$ & $<.04$ & $<.06$ & $0.47 \quad 2$ & $<.95$ & \\
\hline $23 \mathrm{e}$ & $\mathrm{Sm}$ & $<.04$ & $<.06$ & $<.14$ & $<.95$ & \\
\hline 24 & & $<.04$ & $<.06$ & $<.15$ & $<1.33$ & Not detected. \\
\hline $25 a$ & $\mathrm{SBc}$ & $<.04$ & $<.07$ & $\begin{array}{ll}0.60 & 3\end{array}$ & $\begin{array}{ll}1.49 \quad 2\end{array}$ & \\
\hline $25 \mathrm{~b}$ & $\mathrm{SBa}$ & $<.04$ & $<.07$ & $<.11$ & $<1.24$ & \\
\hline $25 \mathrm{c}$ & $\mathrm{Sb}$ & $<.04$ & $<.07$ & 0.70 (ce) 3 & 1.55 (ce) 32 & \\
\hline $25 \mathrm{~d}$ & S0 & $<.04$ & $<.07$ & $<.11$ & $<1.24$ & \\
\hline $25 \mathrm{e}$ & $\mathrm{Sdm}$ & $<.04$ & $<.07$ & & & \\
\hline $25 f$ & S0 & $<.04$ & $<.07$ & $<.11$ & $<1.24$ & \\
\hline $25 \mathrm{~g}$ & S0 & $<.04$ & $<.07$ & $<.11$ & $<1.24$ & \\
\hline $26 a$ & Scd & $<.03$ & $<.05$ & 0.54 (abdg) 3 & 1.496 (abdg) 3 & \\
\hline $26 \mathrm{~b}$ & E0 & $<.03$ & $<.05$ & & & \\
\hline $26 \mathrm{c}$ & S0 & $<.03$ & $<.05$ & $<.09$ & $<.62$ & \\
\hline $26 \mathrm{~d}$ & $\mathrm{cI}$ & $<.03$ & $<.05$ & & & \\
\hline $26 \mathrm{e}$ & $\operatorname{Im}$ & $<.03$ & $<.05$ & $<.09$ & $<.62$ & \\
\hline $26 f$ & $\mathrm{cI}$ & $<.03$ & $<.05$ & $<.09$ & $<.62$ & \\
\hline $26 \mathrm{~g}$ & So & $<.03$ & $<.05$ & & & \\
\hline $27 \mathrm{a}$ & $\mathrm{Sb}$ & $<.04$ & $<.05$ & $<.15$ & $<.17$ & \\
\hline $27 \mathrm{~b}$ & $\mathrm{SBc}$ & $<.04$ & $<.05$ & 0.27 (bdf) 3 & 0.75 (bdf) 2 & \\
\hline $27 \mathrm{c}$ & $\mathrm{Sa}$ & $<.04$ & $<.05$ & $<.15$ & $<.17$ & \\
\hline $27 \mathrm{~d}$ & So & $<.04$ & $<.05$ & & & \\
\hline $27 \mathrm{e}$ & So & $<.04$ & $<.05$ & $<.15$ & $<.17$ & \\
\hline $27 \mathrm{f}$ & - & $<.04$ & $<.05$ & & & \\
\hline 28 & & $<.05$ & $<.06$ & $<.23$ & $<1.37$ & Not detected. \\
\hline $29 \mathrm{a}$ & $\mathrm{cI}$ & $<.04$ & $<.05$ & 0.22 (abcd) 3 & $<.86$ & \\
\hline $29 b$ & So & $<.04$ & $<.05$ & & $<.86$ & \\
\hline $29 \mathrm{c}$ & $\mathrm{E} 2$ & $<.04$ & $<.05$ & & $<.86$ & \\
\hline $29 \mathrm{~d}$ & SB0 & $<.04$ & $<.05$ & & $<.86$ & \\
\hline 30 & & $<.03$ & $<.05$ & $<.15$ & $<.70$ & Not detected; P.O.s available \\
\hline $31 \mathrm{a}$ & $\mathrm{Sdm}$ & $<.04$ & $0.58(\mathrm{ac}) 3$ & 4.06 (abcd) 3 & 5.64 (abcd) 2 & \\
\hline $31 b$ & $\mathrm{Sm}$ & $<.04$ & $<.06$ & & & \\
\hline $31 \mathrm{c}$ & $\operatorname{Im}$ & $<.04$ & & & & \\
\hline $31 d$ & $\mathrm{Sbc}$ & $<.04$ & $<.06$ & & & \\
\hline 32 & & $<.03$ & $<.05$ & $<.21$ & $<1.67$ & Not detected; P.O.s available \\
\hline $33 \mathrm{a}$ & E1 & $<.05$ & $<.09$ & $<.49$ & $<1.14$ & \\
\hline $33 \mathrm{~b}$ & $\mathrm{E} 4$ & $<.05$ & $<.09$ & $<.49$ & $<1.14$ & \\
\hline $33 \mathrm{c}$ & $\mathrm{Sd}$ & $<.05$ & $<.09$ & $0.66 \quad 2$ & $<1.14$ & \\
\hline $33 \mathrm{~d}$ & E0 & $<.05$ & $<.09$ & $<.49$ & $<1.14$ & \\
\hline
\end{tabular}


Table 2. continued

\begin{tabular}{|c|c|c|c|c|c|c|c|}
\hline Ident. & $\mathrm{T}$ & $12 \mu \mathrm{m}$ & $25 \mu \mathrm{m}$ & & $60 \mu \mathrm{m}$ & $100 \mu \mathrm{m}$ & Comments \\
\hline $34 \mathrm{a}$ & E2 & $<.08$ & $<.13$ & & $<.09$ & $<.35$ & \\
\hline $34 \mathrm{~b}$ & $\mathrm{Sd}$ & $<.08$ & $<.13$ & & $1.35(\mathrm{bcd}) \quad 3$ & 0.42 (bcd) 3 & \\
\hline $34 \mathrm{c}$ & SBd & $<.08$ & $<.13$ & & & & \\
\hline $34 d$ & S0 & $<.08$ & $<.13$ & & & & \\
\hline 35 & & $<.04$ & $<.05$ & & $<.08$ & $<.34$ & Not detected. \\
\hline $36 \mathrm{a}$ & $\mathrm{Sb}$ & $<.06$ & $<.11$ & & $0.30 \quad 1$ & 0.83 (abcd) 3 & \\
\hline $36 \mathrm{~b}$ & $\mathrm{Sc}$ & $<.06$ & $<.11$ & & $<.26$ & & \\
\hline $36 \mathrm{c}$ & Scd & $<.06$ & $<.11$ & & $<.26$ & & \\
\hline $36 \mathrm{~d}$ & $\mathrm{Sbc}$ & $<.06$ & $<.11$ & & $<.26$ & & \\
\hline $37 \mathrm{a}$ & E7 & $<.05$ & $<.09$ & & $<.11$ & $<.30$ & P.O.s available. \\
\hline $37 \mathrm{~b}$ & $\mathrm{Sbc}$ & $<.05$ & $<.09$ & & $0.37 \quad 3$ & $2.00(\mathrm{bcd}) \quad 3$ & \\
\hline $37 \mathrm{c}$ & S0a & $<.05$ & $<.09$ & & 0.192 & & \\
\hline $37 d$ & SBdm & $<.05$ & $<.09$ & & $<.11$ & & \\
\hline $37 \mathrm{e}$ & E0 & $<.05$ & $<.09$ & & $<.11$ & $<.30$ & \\
\hline $38 \mathrm{a}$ & Sbc & $<.05$ & $<.07$ & & $<.10$ & $<.44$ & \\
\hline $38 \mathrm{~b}$ & $\mathrm{SBd}$ & $<.05$ & $0.45(\mathrm{bc})$ & 2 & $1.43(\mathrm{bc}) \quad 3$ & 3.16 (bc) 3 & \\
\hline $38 \mathrm{c}$ & $\operatorname{Im}$ & $<.05$ & & & & & \\
\hline $38 \mathrm{~d}$ & $\mathrm{SBa}$ & $<.05$ & $<.07$ & & $<.10$ & $<.44$ & \\
\hline $39 a$ & $\mathrm{Sb}$ & $<.05$ & $<.07$ & & 0.45 (abcd) 3 & $<.44$ & \\
\hline $39 b$ & So & $<.05$ & $<.07$ & & & $<.44$ & \\
\hline $39 \mathrm{c}$ & $\mathrm{Sc}$ & $<.05$ & $<.07$ & & & $<.44$ & \\
\hline $39 d$ & So & $<.05$ & $<.07$ & & & $<.44$ & \\
\hline $40 \mathrm{a}$ & E3 & $<.05$ & $<.06$ & & $<.13$ & 3.85 (abcde) 3 & P.O.s available. \\
\hline $40 \mathrm{~b}$ & S0 & $<.05$ & $<.06$ & & 0.67 (bc) 3 & & \\
\hline $40 \mathrm{c}$ & $\mathrm{Sbc}$ & $<.05$ & $<.06$ & & & & \\
\hline $40 \mathrm{~d}$ & Sba & $<.05$ & $<.06$ & & $<.13$ & & \\
\hline $40 \mathrm{e}$ & $\mathrm{Sc}$ & $<.05$ & $<.06$ & & $0.42 \quad 3$ & & \\
\hline $41 \mathrm{a}$ & Sab & $<.04$ & $<.05$ & & $0.14 \quad 3$ & $1.2(\mathrm{abc}) 2$ & P.O.s available. \\
\hline $41 \mathrm{~b}$ & $\mathrm{Sab}$ & $<.04$ & $<.05$ & & $<.06$ & & \\
\hline $41 \mathrm{c}$ & $\mathrm{Sb}$ & $<.04$ & $<.05$ & & $<.07$ & & \\
\hline $41 d$ & So & $<.04$ & $<.05$ & & $<.06$ & $<.30$ & \\
\hline 42 & & & & & & & Not observed by IRAS. \\
\hline $43 a$ & $\mathrm{Sb}$ & $0.27 \quad 3$ & $<.09$ & & 0.28 (af) 3 & $1.58 \quad 3$ & \\
\hline $43 \mathrm{~b}$ & SBcd & $<.04$ & $<.09$ & & $0.27 \quad 2$ & $1.27 \quad 2$ & \\
\hline $43 \mathrm{c}$ & SB0 & $<.04$ & $<.09$ & & $<.12$ & $<.61$ & \\
\hline $43 \mathrm{~d}$ & $\mathrm{Sc}$ & $<.04$ & $<.09$ & & $<.12$ & $<.61$ & \\
\hline $43 \mathrm{e}$ & So & $<.04$ & $<.09$ & & $<.12$ & $<.61$ & \\
\hline $43 \mathrm{f}$ & $\mathrm{Sbc}$ & $<.04$ & $<.09$ & & & $<.61$ & \\
\hline $44 \mathrm{a}$ & $\mathrm{Sa}$ & $0.32 \quad 2$ & $0.35 \quad 3$ & & 3.193 & $8.87 \quad 3$ & P.O.s available. \\
\hline $44 \mathrm{~b}$ & $\mathrm{E} 2$ & $<.05$ & $<.10$ & & $<.16$ & $<.36$ & \\
\hline $44 \mathrm{c}$ & $\mathrm{SBc}$ & $<.05$ & $<.10$ & & $1.43 \quad 3$ & $3.68 \quad 3$ & \\
\hline $44 d$ & $\mathrm{Sd}$ & $<.05$ & $<.10$ & & $0.91 \quad 3$ & $1.41 \quad 2$ & \\
\hline $45 \mathrm{a}$ & $\mathrm{Sa}$ & $<.06$ & $<.05$ & & $0.21(\mathrm{ad}) \quad 1$ & $1.51(\mathrm{ad}) \quad 2$ & \\
\hline $45 \mathrm{~b}$ & S0a & $<.06$ & $<.05$ & & $<.09$ & $<.29$ & \\
\hline $45 \mathrm{c}$ & $\mathrm{Sc}$ & $<.06$ & $<.05$ & & $<.09$ & $<.29$ & \\
\hline $45 \mathrm{~d}$ & So & $<.06$ & $<.05$ & & & & \\
\hline 46 & & $<.07$ & $<.14$ & & $<.10$ & $<.34$ & Not detected; P.O.s available. \\
\hline $47 \mathrm{a}$ & $\mathrm{SBb}$ & $<.05$ & $<.11$ & & $0.58 \quad 3$ & $1.51 \quad 3$ & \\
\hline $47 \mathrm{~b}$ & E3 & $<.05$ & $<.11$ & & $<.23$ & $<.55$ & \\
\hline $47 \mathrm{c}$ & $\mathrm{Sc}$ & $<.05$ & $<.11$ & & $<.23$ & $<.55$ & \\
\hline $47 \mathrm{~d}$ & $\mathrm{Sd}$ & $<.05$ & $<.11$ & & $<.23$ & $<.55$ & \\
\hline $48 \mathrm{a}$ & $\mathrm{E} 2$ & $<.05$ & $<.06$ & & $<.16$ & $<.85$ & P.O.s available. \\
\hline $48 \mathrm{~b}$ & $\mathrm{Sc}$ & $<.05$ & $<.06$ & & $0.80 \quad 2$ & 1.952 & \\
\hline $48 \mathrm{c}$ & $\mathrm{Sc}$ & $<.05$ & $<.06$ & & $<.16$ & $<.85$ & \\
\hline $48 \mathrm{~d}$ & $\mathrm{~S} 0 \mathrm{a}$ & $<.05$ & $<.06$ & & $<.16$ & $<.85$ & \\
\hline
\end{tabular}


Table 2. continued

\begin{tabular}{|c|c|c|c|c|c|c|}
\hline Ident. & $\mathrm{T}$ & $12 \mu \mathrm{m}$ & $25 \mu \mathrm{m}$ & $60 \mu \mathrm{m}$ & $100 \mu \mathrm{m}$ & Comments \\
\hline $49 \mathrm{a}$ & Scd & $<.03$ & $<.04$ & 0.44 (abcd) 3 & 0.73 (abcd) 2 & \\
\hline $49 \mathrm{~b}$ & $\mathrm{Sd}$ & $<.03$ & $<.04$ & & & \\
\hline $49 \mathrm{c}$ & $\operatorname{Im}$ & $<.03$ & $<.04$ & & & \\
\hline $49 \mathrm{~d}$ & E3 & $<.03$ & $<.04$ & & & \\
\hline 50 & & $<.04$ & $<.05$ & $<.10$ & $<.34$ & Not detected. \\
\hline 51 & & & & & & Not observed by IRAS. \\
\hline 52 & & & & & & Not observed by IRAS. \\
\hline 53 & & $<.06$ & $<.10$ & $<.11$ & $<.40$ & Not detected. \\
\hline $54 a$ & $\mathrm{Sdm}$ & $<.06$ & $<.10$ & 0.38 (abcd) 3 & 0.71 (abcd) 2 & \\
\hline $54 \mathrm{~b}$ & $\operatorname{Im}$ & $<.06$ & $<.10$ & & & \\
\hline $54 \mathrm{c}$ & $\operatorname{Im}$ & $<.06$ & $<.10$ & & & \\
\hline $54 \mathrm{~d}$ & $\operatorname{Im}$ & $<.06$ & $<.10$ & & & \\
\hline $55 \mathrm{a}$ & E0 & $<.04$ & $<.04$ & 0.20 (abcde) 2 & 1.63 (abcde) 3 & P.O.s available. \\
\hline $55 \mathrm{~b}$ & S0 & $<.04$ & $<.04$ & & & \\
\hline $55 \mathrm{c}$ & E3 & $<.04$ & $<.04$ & & & \\
\hline $55 \mathrm{~d}$ & E2 & $<.04$ & $<.04$ & & & \\
\hline $55 \mathrm{e}$ & $\mathrm{Sc}$ & $<.04$ & $<.04$ & & & \\
\hline $56 \mathrm{a}$ & $\mathrm{Sc}$ & $<.04$ & $<.05$ & $<.10$ & $<.38$ & P.O.s available. \\
\hline $56 \mathrm{~b}$ & SB0 & 0.17 & 0.24 & $0.69(\mathrm{bcd}) \quad 3$ & $1.51(\mathrm{bcd}) \quad 3$ & \\
\hline $56 \mathrm{c}$ & S0 & $<.04$ & $<.05$ & & & \\
\hline $56 \mathrm{~d}$ & S0 & $<.04$ & $<.05$ & & & \\
\hline $56 \mathrm{e}$ & S0 & $<.04$ & $<.05$ & $<.10$ & $<.38$ & \\
\hline $57 \mathrm{a}$ & $\mathrm{Sb}$ & $<.06$ & $<.09$ & $0.46(\mathrm{ad}) \quad 3$ & $1.38(\mathrm{ad}) \quad 3$ & \\
\hline $57 \mathrm{~b}$ & $\mathrm{SBb}$ & $<.06$ & $<.09$ & $<.14$ & $<.36$ & \\
\hline $57 \mathrm{c}$ & E3 & $<.06$ & $<.09$ & $<.14$ & $<.36$ & \\
\hline $57 \mathrm{~d}$ & $\mathrm{SBc}$ & $<.06$ & $<.09$ & & & \\
\hline $57 \mathrm{e}$ & $\mathrm{S} 0 \mathrm{a}$ & $<.06$ & $<.09$ & $<.14$ & $<.36$ & \\
\hline $57 \mathrm{f}$ & E4 & $<.06$ & $<.09$ & $<.14$ & $<.36$ & \\
\hline $57 \mathrm{~g}$ & SB0 & $<.06$ & $<.09$ & $<.14$ & $<.36$ & \\
\hline $57 \mathrm{~h}$ & $\mathrm{SBb}$ & $<.06$ & $<.09$ & $<.14$ & $<.36$ & \\
\hline 58 & & $<.06$ & $<.09$ & $<.15$ & $<.53$ & Not detected. \\
\hline $59 \mathrm{a}$ & $\mathrm{Sa}$ & $<.06$ & $<.08$ & $<.10$ & $3.81(\mathrm{ad}) \quad 3$ & \\
\hline $59 \mathrm{~b}$ & E0 & $<.06$ & $<.08$ & $<.10$ & $<.34$ & \\
\hline $59 \mathrm{c}$ & $\mathrm{Sc}$ & $<.06$ & $<.08$ & $<.10$ & $<.34$ & \\
\hline $59 \mathrm{~d}$ & $\operatorname{Im}$ & $<.06$ & $0.59 \quad 2$ & $3.58 \quad 3$ & & \\
\hline $59 \mathrm{e}$ & Scd & $<.06$ & $<.08$ & $<.10$ & $<.34$ & \\
\hline 60 & & $<.04$ & $<.05$ & $<.11$ & $<.46$ & Not detected. \\
\hline $61 \mathrm{a}$ & S0a & $<.05$ & $<.06$ & $<.13$ & $<.44$ & \\
\hline $61 b$ & $\operatorname{Im}$ & $<.05$ & $<.06$ & $1.13 \quad 3$ & $<.44$ & \\
\hline $61 \mathrm{c}$ & $\mathrm{Sbc}$ & $0.26 \quad 2$ & $0.52 \quad 2$ & $5.00 \quad 3$ & $11.13 \quad 3$ & \\
\hline $61 d$ & S0 & $<.05$ & $<.06$ & $<.13$ & $<.44$ & \\
\hline 62 & & $<.06$ & $<.10$ & $<.13$ & $<.72$ & Not detected. \\
\hline $63 a$ & SBbc & $<.02$ & $<.08$ & $\begin{array}{ll}0.36 & 2\end{array}$ & $1.10 \quad 3$ & \\
\hline $63 \mathrm{~b}$ & $\mathrm{SBc}$ & $<.02$ & $<.08$ & $<.19$ & $<.95$ & \\
\hline $63 \mathrm{c}$ & $\mathrm{SBc}$ & $<.02$ & $<.08$ & $<.19$ & $<.95$ & \\
\hline $63 \mathrm{~d}$ & $\mathrm{Sc}$ & $<.02$ & $<.08$ & $0.94 \quad 3$ & $2.13 \quad 3$ & \\
\hline 64 & & $<.05$ & $<.11$ & $<.19$ & $<.63$ & Not detected. \\
\hline 65 & & $<.05$ & $<.07$ & $<.13$ & $<.65$ & Not detected; P.O.s available. \\
\hline 66 & & $<.03$ & $<.03$ & $<.13$ & $<.30$ & Not detected; P.O.s available. \\
\hline $67 a$ & E1 & $<.06$ & $<.10$ & $<.46$ & $<.80$ & \\
\hline $67 \mathrm{~b}$ & Sc & $<.06$ & $<.10$ & $0.88 \quad 2$ & $2.91 \quad 3$ & \\
\hline $67 \mathrm{c}$ & Scd & $<.06$ & $<.10$ & $<.46$ & $<.80$ & \\
\hline $67 \mathrm{~d}$ & S0 & $<.06$ & $<.10$ & $<.46$ & $<.80$ & \\
\hline
\end{tabular}


Table 2. continued

\begin{tabular}{|c|c|c|c|c|c|c|c|c|}
\hline Ident. & $\mathrm{T}$ & $12 \mu \mathrm{m}$ & $25 \mu \mathrm{m}$ & & $60 \mu \mathrm{m}$ & & $100 \mu \mathrm{m}$ & Comments \\
\hline $68 \mathrm{a}$ & S0 & $<.04$ & $<.05$ & & 0.121 & & $1.09 \quad 2$ & \\
\hline $68 \mathrm{~b}$ & E2 & $<.04$ & $<.05$ & & $0.18 \quad 2$ & & $0.39 \quad 2$ & \\
\hline $68 c$ & $\mathrm{SBbc}$ & $<.04$ & $1.07 \quad 3$ & & $2.22 \quad 3$ & & 8.613 & \\
\hline $68 \mathrm{~d}$ & E3 & $<.04$ & $<.05$ & & $<.11$ & & $<.36$ & \\
\hline $68 \mathrm{e}$ & S0 & $<.04$ & $<.05$ & & $<.11$ & & $<.36$ & \\
\hline $69 a$ & $\mathrm{Sc}$ & $<.05$ & $<.06$ & & $<.10$ & & $<.29$ & \\
\hline $69 \mathrm{~b}$ & $\mathrm{SBb}$ & $<.05$ & $0.41 \quad 2$ & & $2.19 \quad 3$ & & $2.96 \quad 2$ & \\
\hline $69 c$ & So & $<.05$ & $<.06$ & & $<.10$ & & $<.29$ & \\
\hline $69 \mathrm{~d}$ & SB0 & $<.05$ & $<.06$ & & $<.10$ & & $<.29$ & \\
\hline $70 \mathrm{a}$ & S0a & $<.04$ & $<.05$ & & $<.08$ & & 1.97 (acdefg) 3 & \\
\hline $70 \mathrm{~b}$ & $\mathrm{SBa}$ & $<.04$ & $<.05$ & & $0.38 \quad 2$ & & & \\
\hline $70 \mathrm{c}$ & $\mathrm{Sbc}$ & $<.04$ & $<.05$ & & 0.112 & & & \\
\hline $70 \mathrm{~d}$ & $\mathrm{Sc}$ & $<.04$ & $<.05$ & & $<.08$ & & & \\
\hline $70 \mathrm{e}$ & $\mathrm{Sbc}$ & $<.04$ & $<.05$ & & $<.08$ & & & \\
\hline $70 f$ & $\mathrm{SBb}$ & $<.04$ & $<.05$ & & $<.08$ & & & \\
\hline $70 \mathrm{~g}$ & $\mathrm{Sa}$ & $<.04$ & $<.05$ & & $<.08$ & & & \\
\hline $71 \mathrm{a}$ & $\mathrm{SBc}$ & $<.04$ & $<.06$ & & $0.13 \quad 2$ & & $<.31$ & P.O.s available. \\
\hline $71 \mathrm{~b}$ & $\mathrm{Sb}$ & $<.04$ & $<.06$ & & 1.512 & & $3.07 \quad 3$ & \\
\hline $71 \mathrm{c}$ & $\mathrm{SBc}$ & $<.04$ & $<.06$ & & $<.10$ & & $<.31$ & \\
\hline $71 \mathrm{~d}$ & S0 & $<.04$ & $<.06$ & & $<.10$ & & $<.31$ & \\
\hline 72 & & $<.04$ & $<.05$ & & $<.11$ & & $<.53$ & Not detected. \\
\hline $73 \mathrm{a}$ & Scd & $<.04$ & $<.04$ & & $<.10$ & & $\begin{array}{ll}0.89 & 3\end{array}$ & \\
\hline $73 \mathrm{~b}$ & $\operatorname{Im}$ & $0.08 \quad 2$ & $0.33 \quad 3$ & & $0.98 \quad 3$ & & $1.23 \quad 3$ & \\
\hline $73 \mathrm{c}$ & S0 & $<.04$ & $<.04$ & & $<.10$ & & $<.52$ & \\
\hline $73 \mathrm{~d}$ & $\mathrm{Sb}$ & $<.04$ & $<.04$ & & $<.10$ & & $0.55 \quad 3$ & \\
\hline $73 \mathrm{e}$ & $\mathrm{Sd}$ & $<.04$ & $<.04$ & & $<.10$ & & $<.52$ & \\
\hline 74 & & $<.03$ & $<.05$ & & $<.11$ & & $<.53$ & Not detected. \\
\hline $75 a$ & $\mathrm{E} 4$ & $<.03$ & $<.05$ & & $<.12$ & & $<.40$ & P.O.s available. \\
\hline $75 \mathrm{~b}$ & $\mathrm{Sb}$ & $<.03$ & $<.05$ & & $<.12$ & & $<.40$ & \\
\hline $75 \mathrm{c}$ & S0 & $<.03$ & $<.05$ & & 0.21 (cde) & 2 & 0.95 (cde) 2 & \\
\hline $75 \mathrm{~d}$ & $\mathrm{Sd}$ & $<.03$ & $<.05$ & & & & & \\
\hline $75 \mathrm{e}$ & $\mathrm{Sa}$ & $<.03$ & $<.05$ & & & & & \\
\hline $75 \mathrm{f}$ & S0 & $<.03$ & $<.05$ & & $<.12$ & & $<.40$ & \\
\hline 76 & & $<.04$ & $<.06$ & & $<.13$ & & $<.68$ & Not detected. \\
\hline $77 \mathrm{a}$ & S0 & $<.04$ & $<.04$ & & $<.12$ & & $<1.05$ & P.O.s available. \\
\hline $77 \mathrm{~b}$ & S0 & $<.04$ & $<.04$ & & $<.12$ & & $<1.05$ & \\
\hline $77 \mathrm{c}$ & $\mathrm{Im}$ & $<.04$ & $0.67(\mathrm{~cd})$ & 2 & $0.19(\mathrm{~cd})$ & 3 & $<1.05$ & \\
\hline $77 \mathrm{~d}$ & $\operatorname{Im}$ & $<.04$ & & & & & $<1.05$ & \\
\hline $78 \mathrm{a}$ & $\mathrm{SBb}$ & $<.04$ & $0.11 \quad 3$ & & $0.81 \quad 3$ & & $2.20 \quad 3$ & \\
\hline $78 \mathrm{~b}$ & S0 & $<.04$ & $<.04$ & & $<.14$ & & $<.71$ & \\
\hline $78 \mathrm{c}$ & S0 & $<.04$ & $<.04$ & & $<.14$ & & $<.71$ & \\
\hline $78 \mathrm{~d}$ & $\mathrm{Sm}$ & $<.04$ & $<.04$ & & $<.14$ & & $<.71$ & \\
\hline $79 \mathrm{a}$ & E0 & $<.04$ & $<.04$ & & $0.96(\mathrm{ad})$ & 3 & 2.32 (ade) 3 & P.O.s available. \\
\hline $79 b$ & S0 & $<.04$ & $<.04$ & & $<.15$ & & $<.74$ & \\
\hline $79 \mathrm{c}$ & S0 & $<.04$ & $<.04$ & & $<.15$ & & $<.74$ & \\
\hline $79 \mathrm{~d}$ & $\mathrm{Sdm}$ & $<.04$ & $<.04$ & & & & & \\
\hline $79 \mathrm{e}$ & Scd & $<.04$ & $<.04$ & & $<.15$ & & & \\
\hline $80 a$ & $\mathrm{Sd}$ & $0.10(\mathrm{ab}) \quad 2$ & $0.16(\mathrm{ab})$ & 3 & $2.31(\mathrm{ab})$ & 3 & $5.12(\mathrm{ab}) 3$ & P.O.s available \\
\hline $80 \mathrm{~b}$ & $\mathrm{Sa}$ & & & & & & & \\
\hline $80 \mathrm{c}$ & $\mathrm{Im}$ & $<.04$ & $<.03$ & & $<.10$ & & $<.41$ & \\
\hline $80 \mathrm{~d}$ & $\mathrm{Im}$ & $<.04$ & $<.03$ & & $<.10$ & & $<.41$ & \\
\hline $81 a$ & $\mathrm{Sc}$ & $<.03$ & $<.05$ & & $0.43 \quad 3$ & & $<1.40$ & \\
\hline $81 b$ & S0 & $<.03$ & $<.05$ & & $<.21$ & & $<1.40$ & \\
\hline $81 c$ & S0 & $<.03$ & $<.05$ & & $<.21$ & & $<1.40$ & \\
\hline $81 \mathrm{~d}$ & $\mathrm{~S} 0 \mathrm{a}$ & $<.03$ & $<.05$ & & $<.21$ & & $<1.40$ & \\
\hline
\end{tabular}



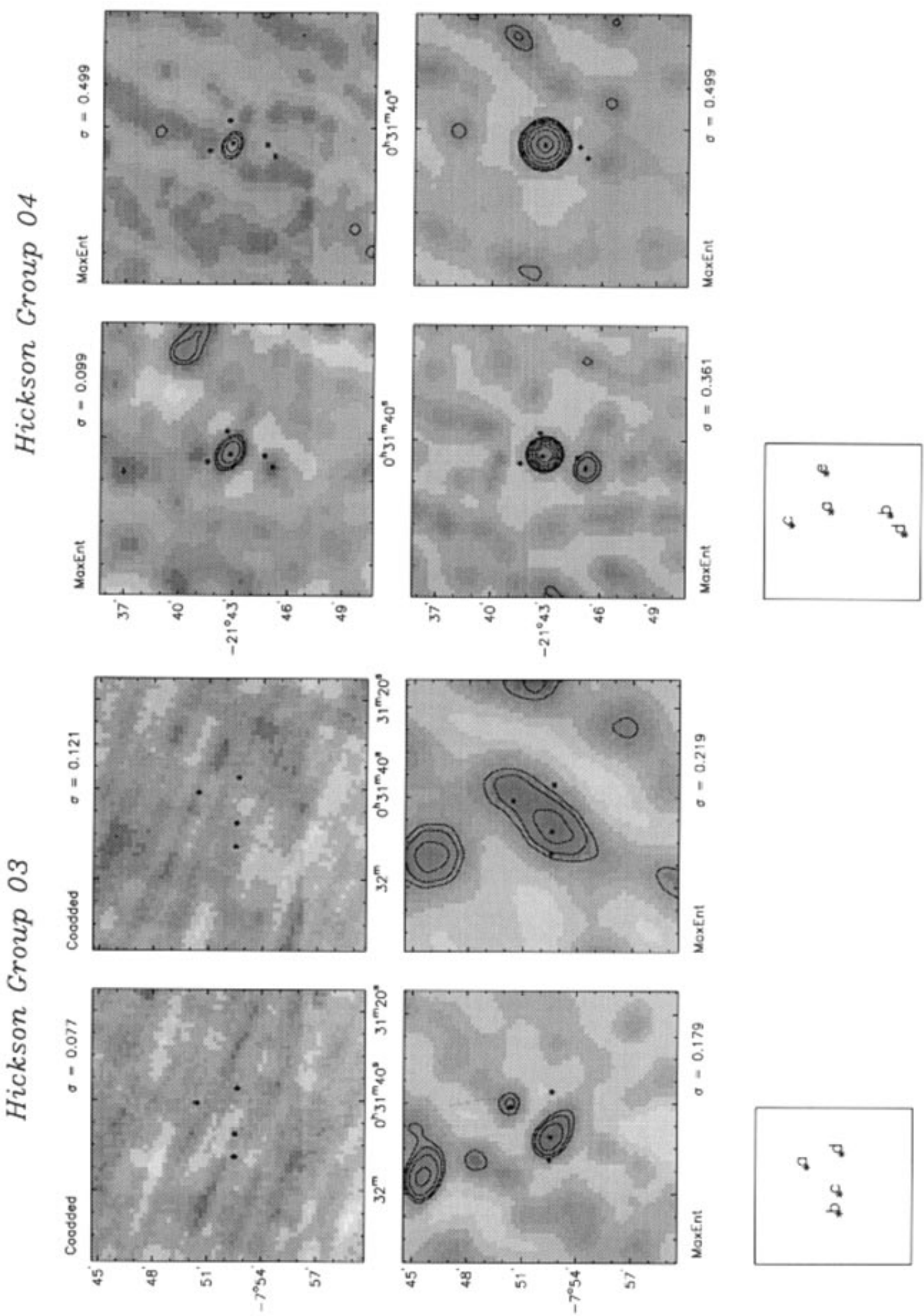

(To be seen in landscape)
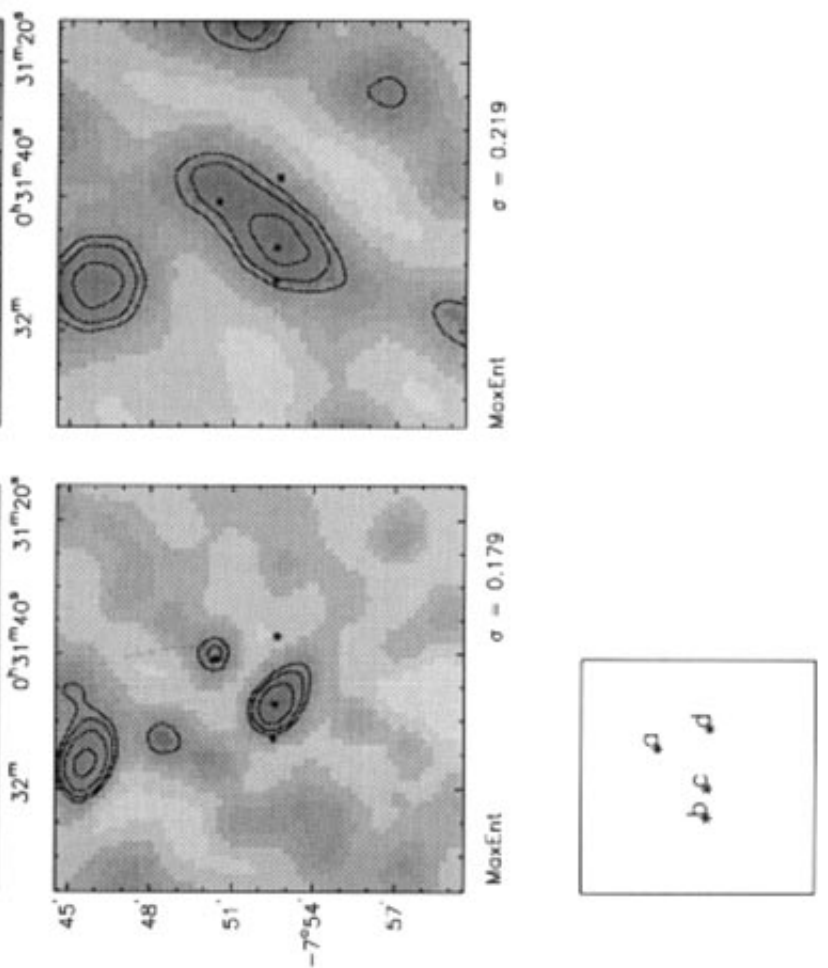

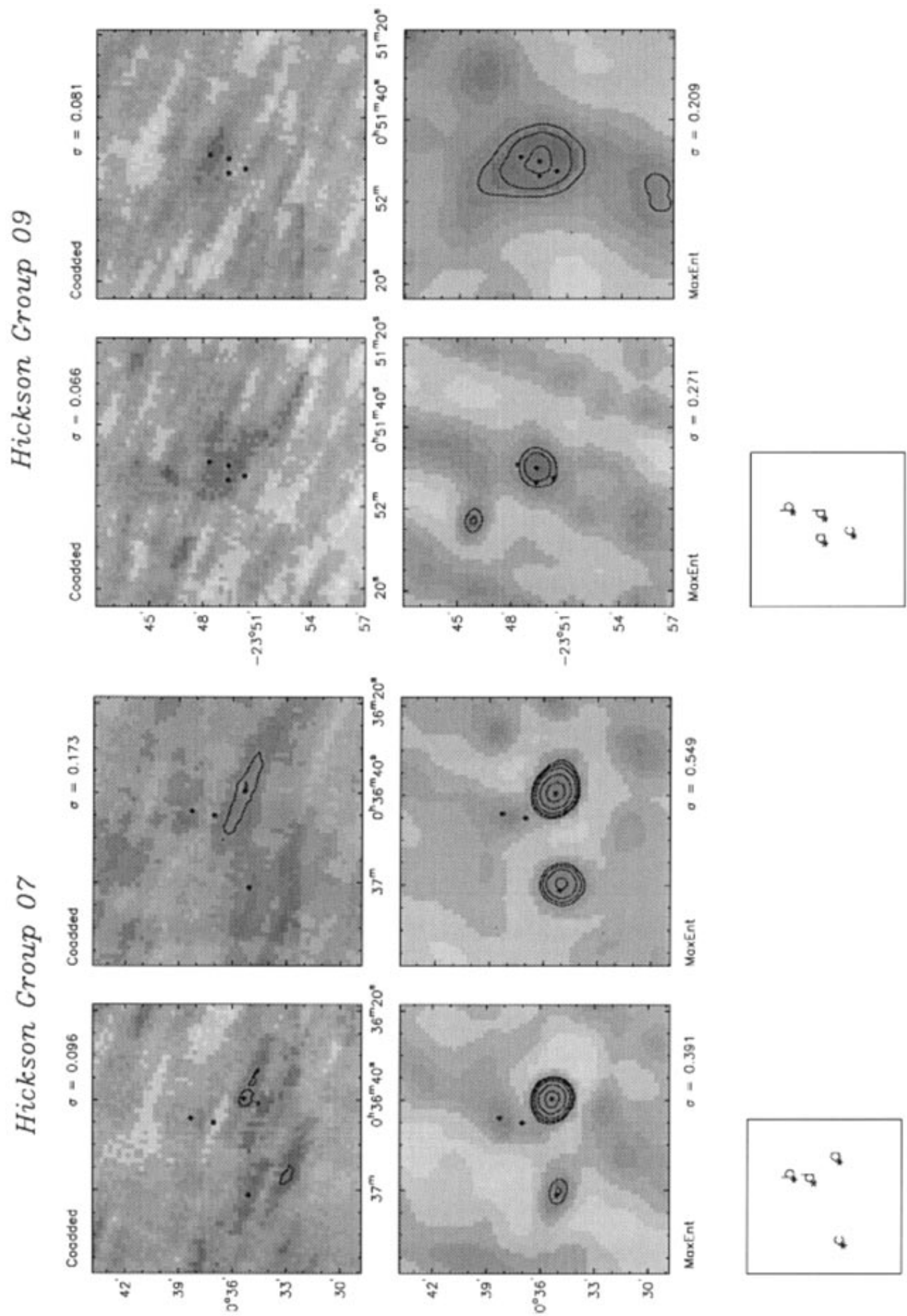

(To be seen in landscape) 

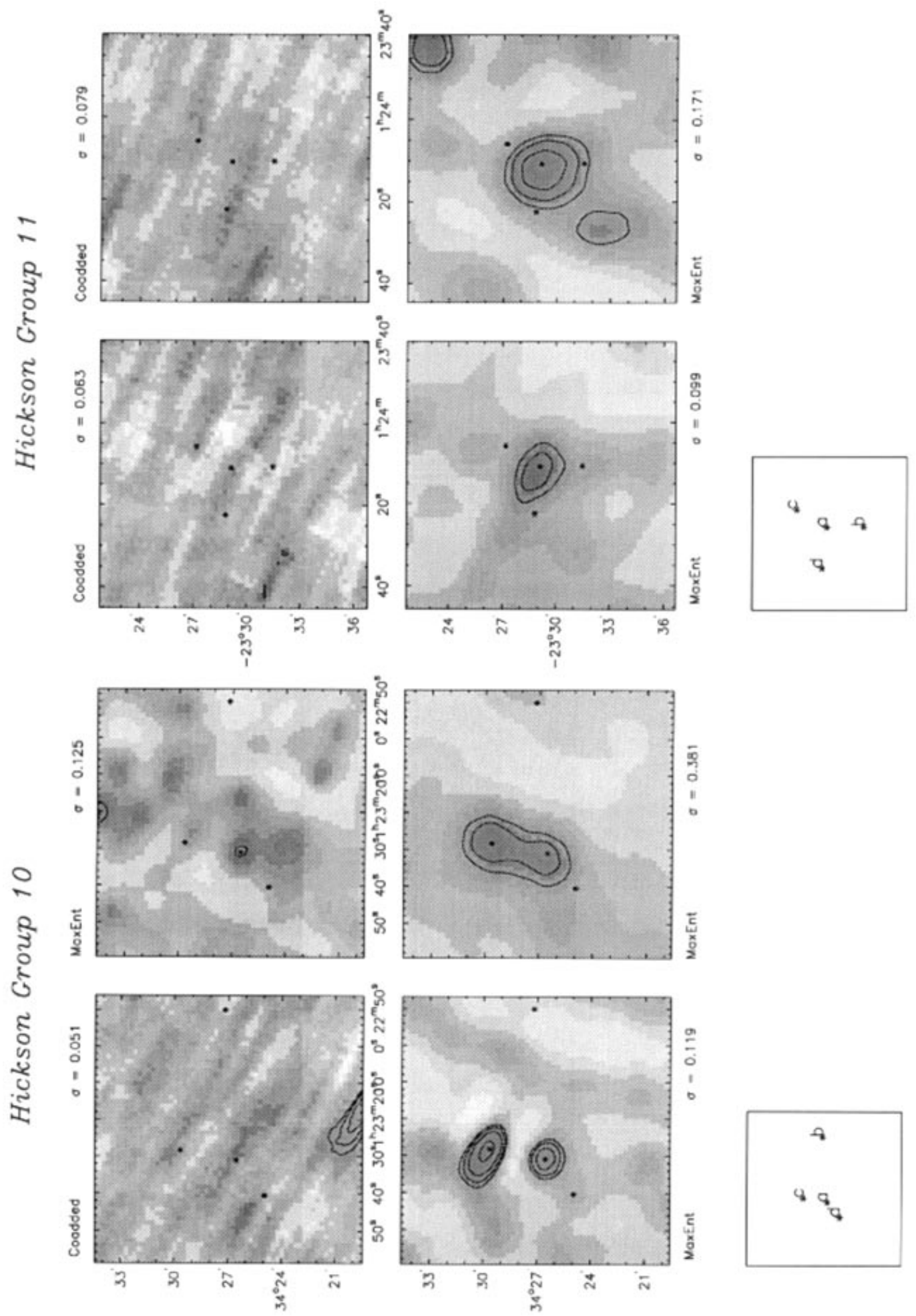

(To be seen in landscape) 

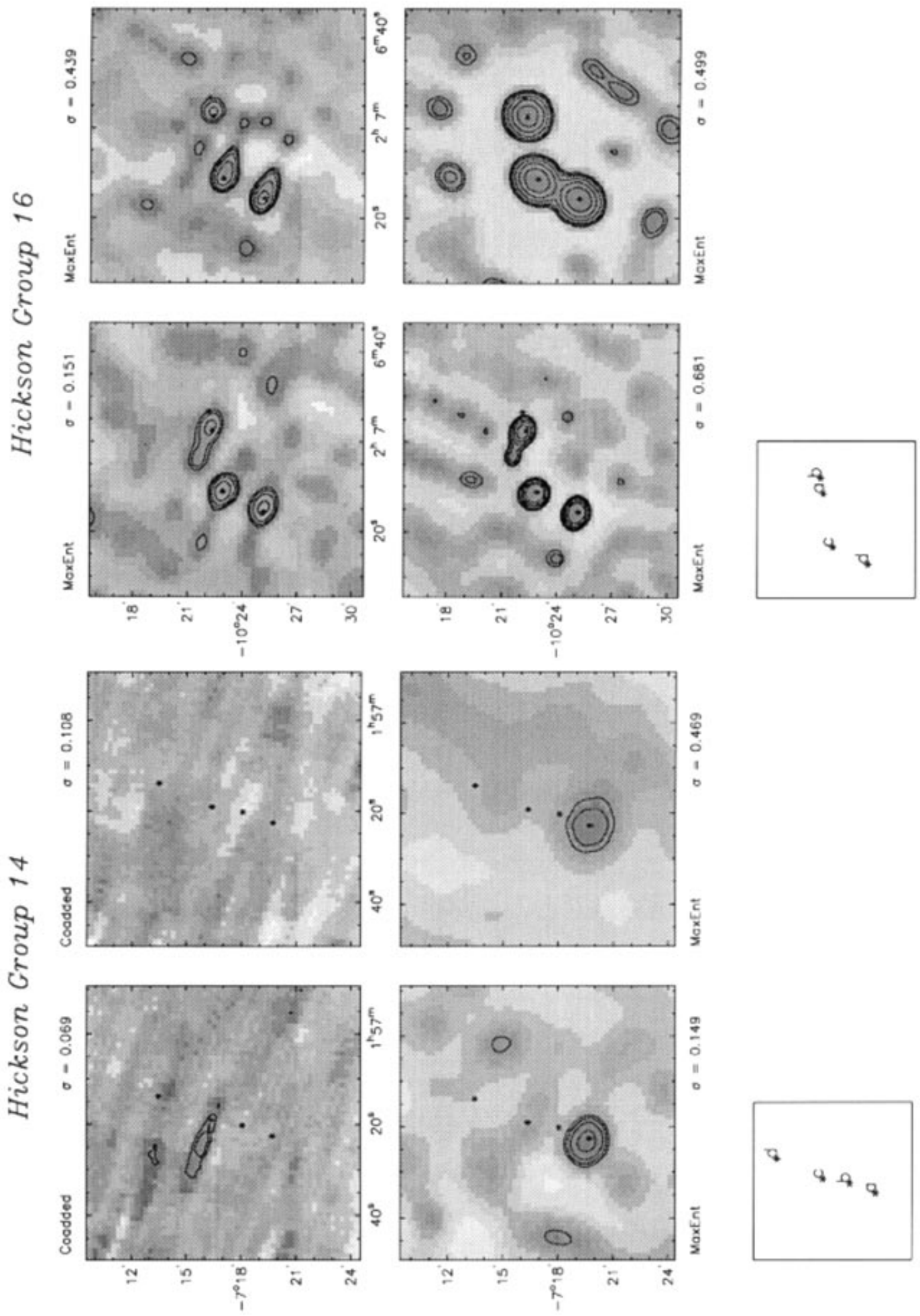

(To be seen in landscape) 

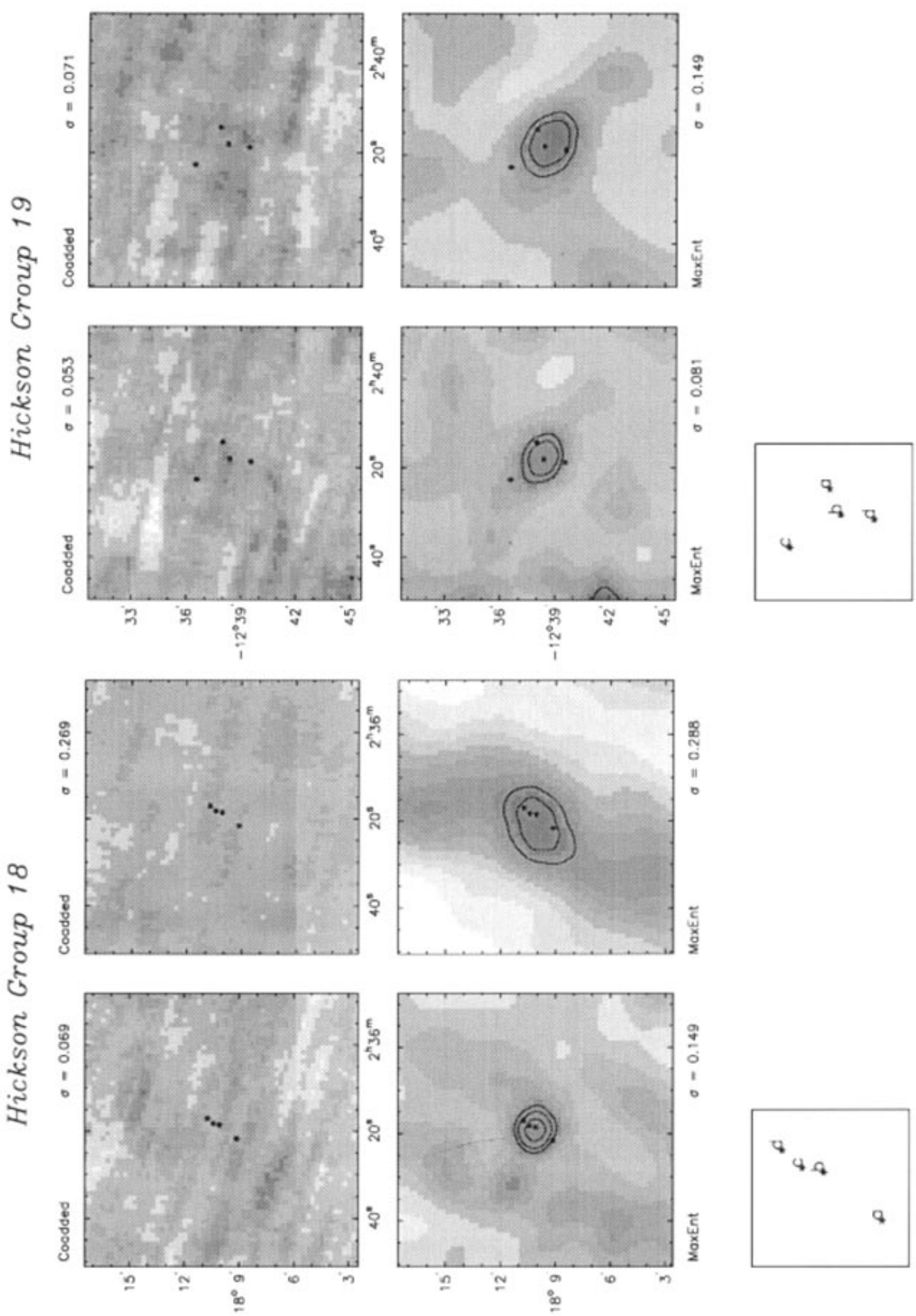

(To be seen in landscape)
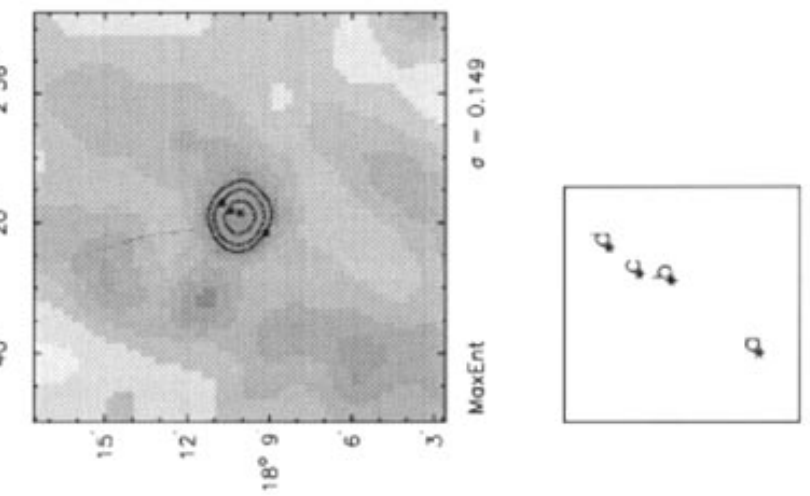


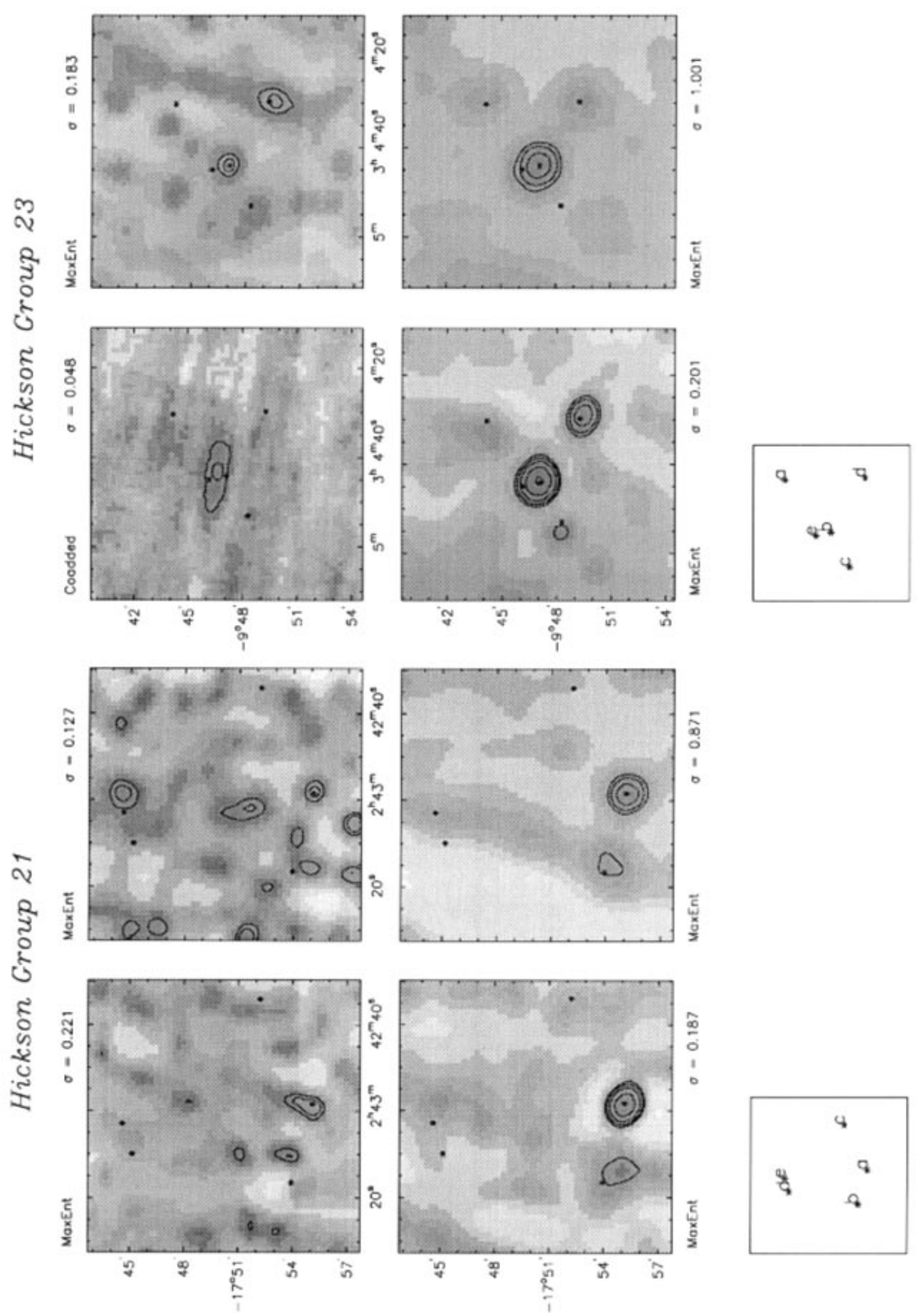

(To be seen in landscape) 

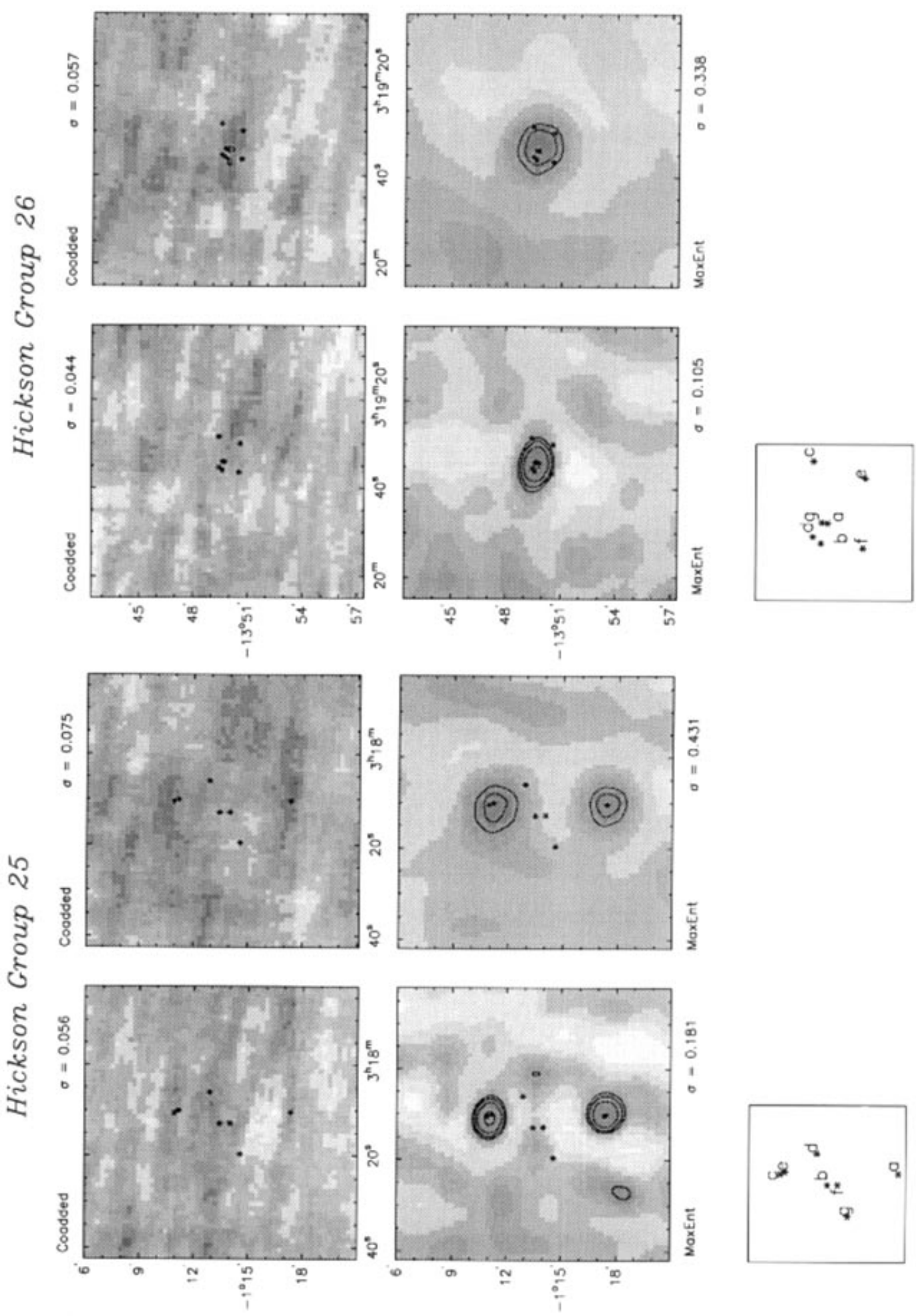

(To be seen in landscape) 

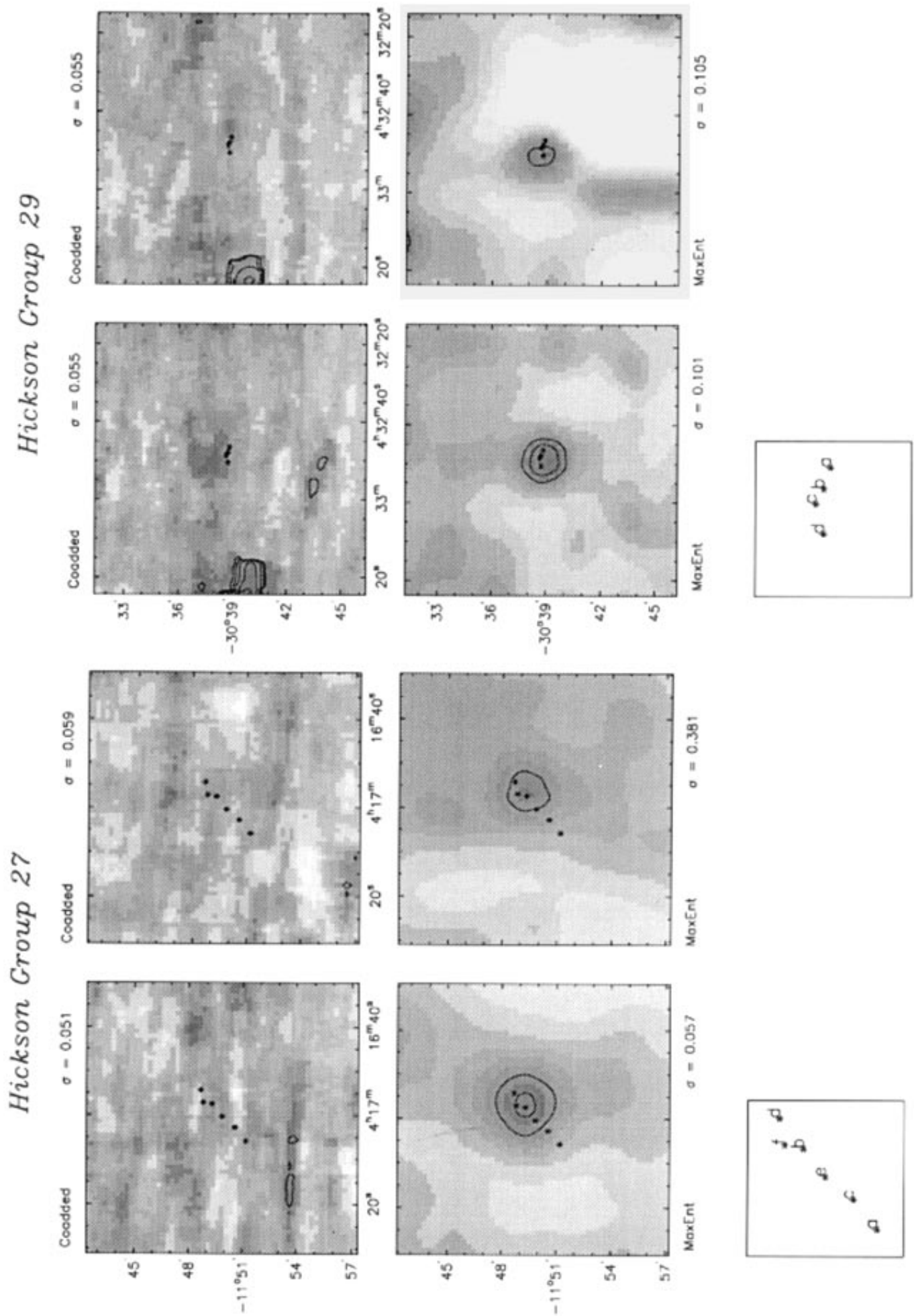

(To be seen in landscape) 

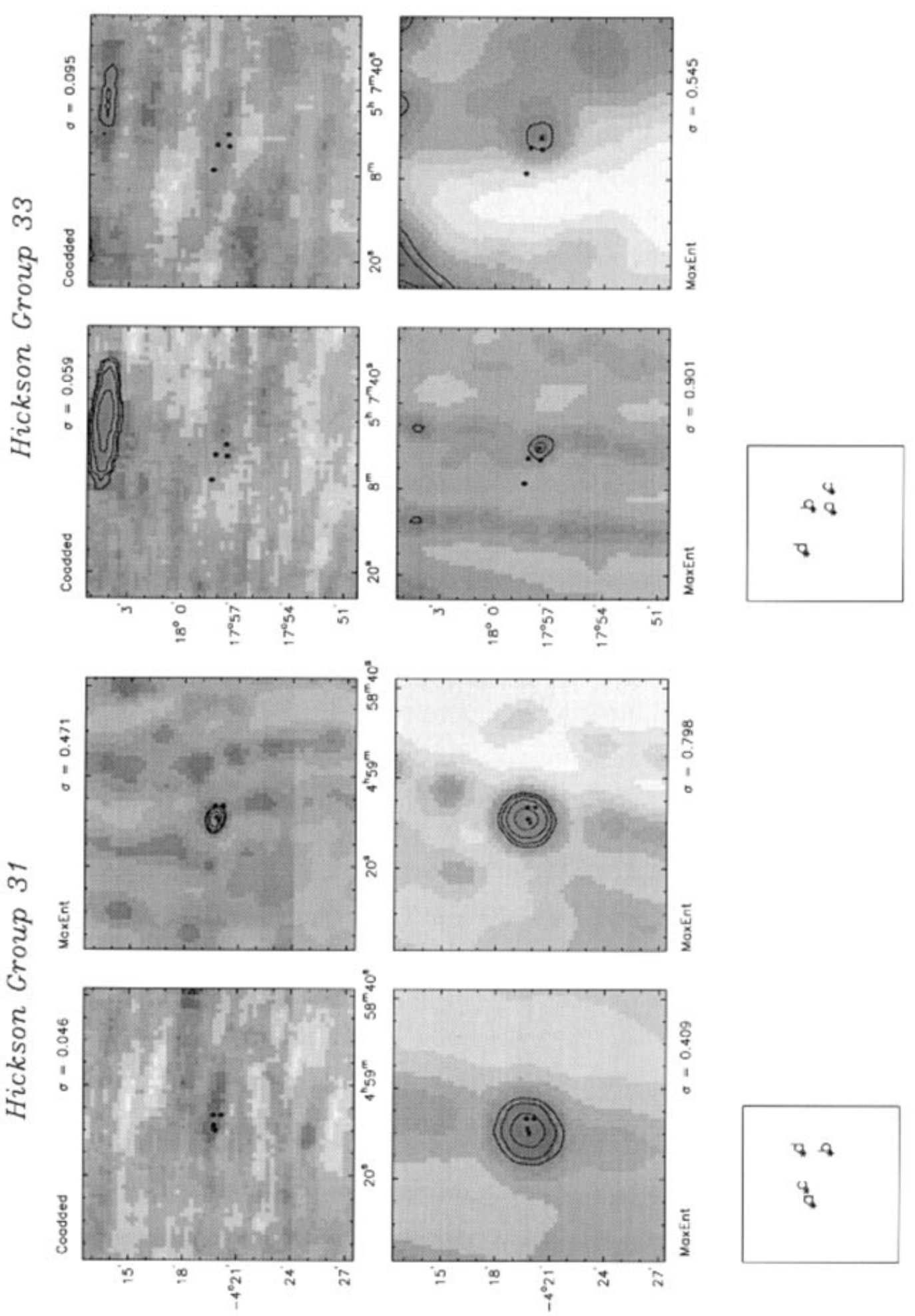

(To be seen in landscape) 

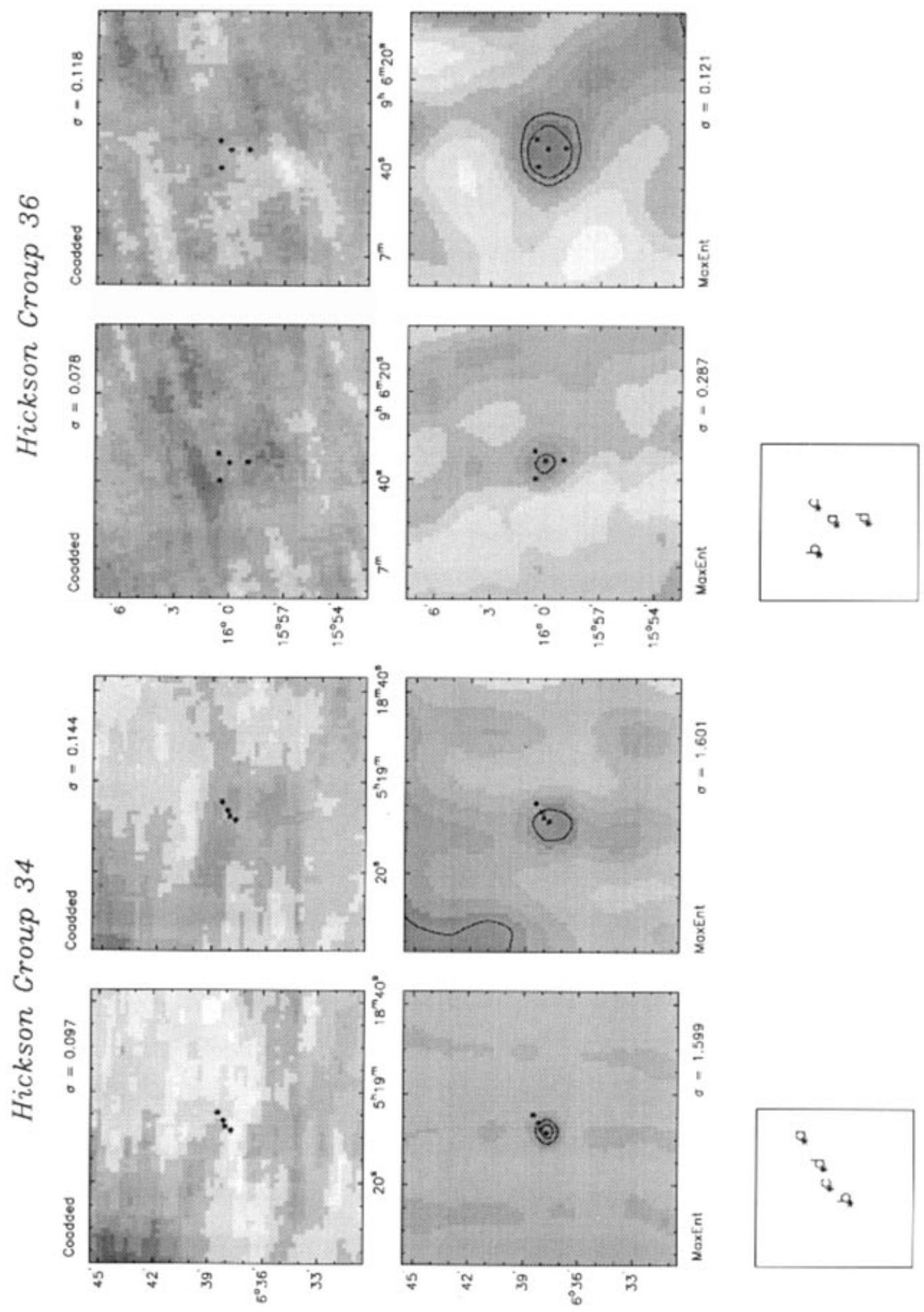

(To be seen in landscape) 

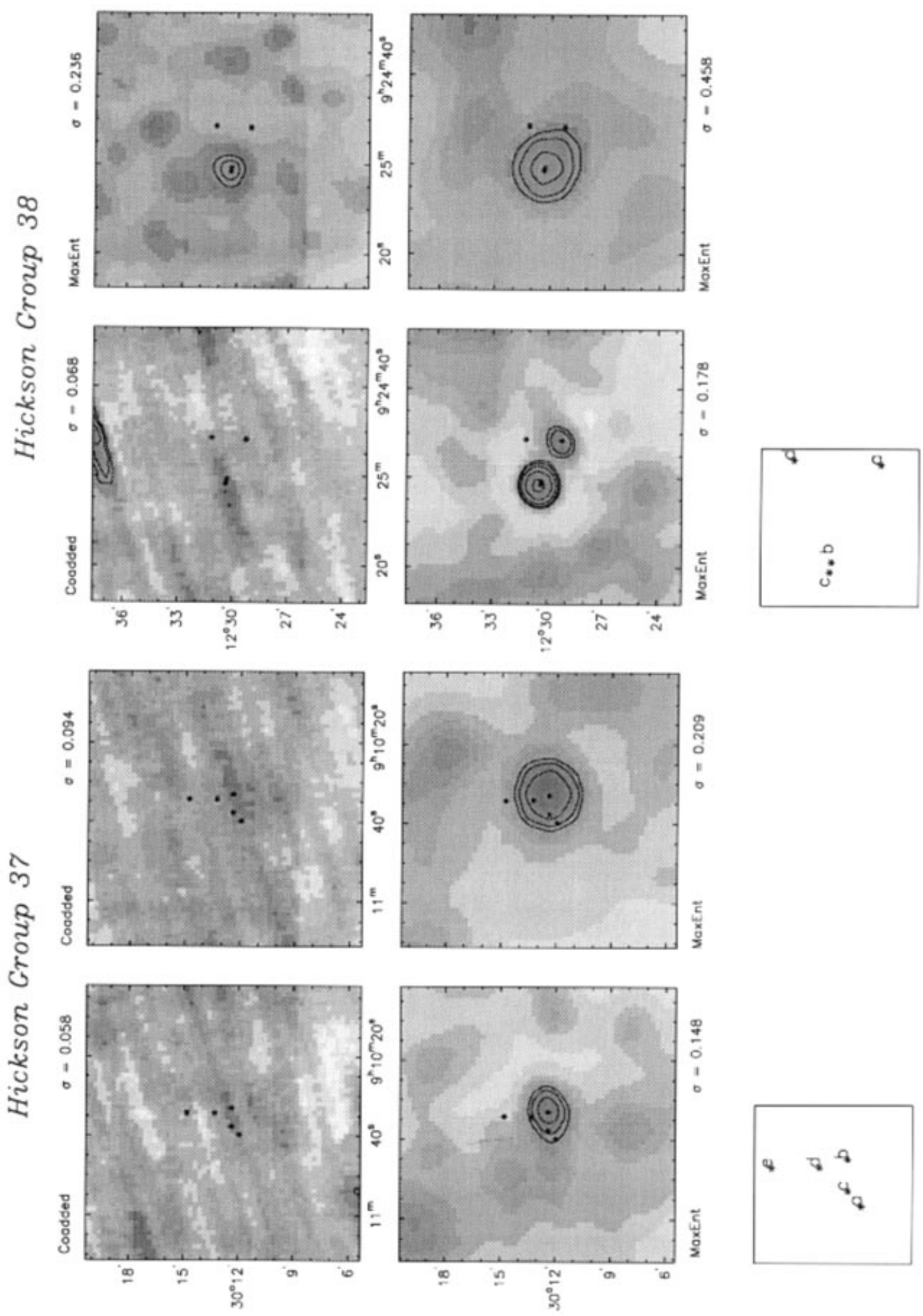

(To be seen in landscape) 

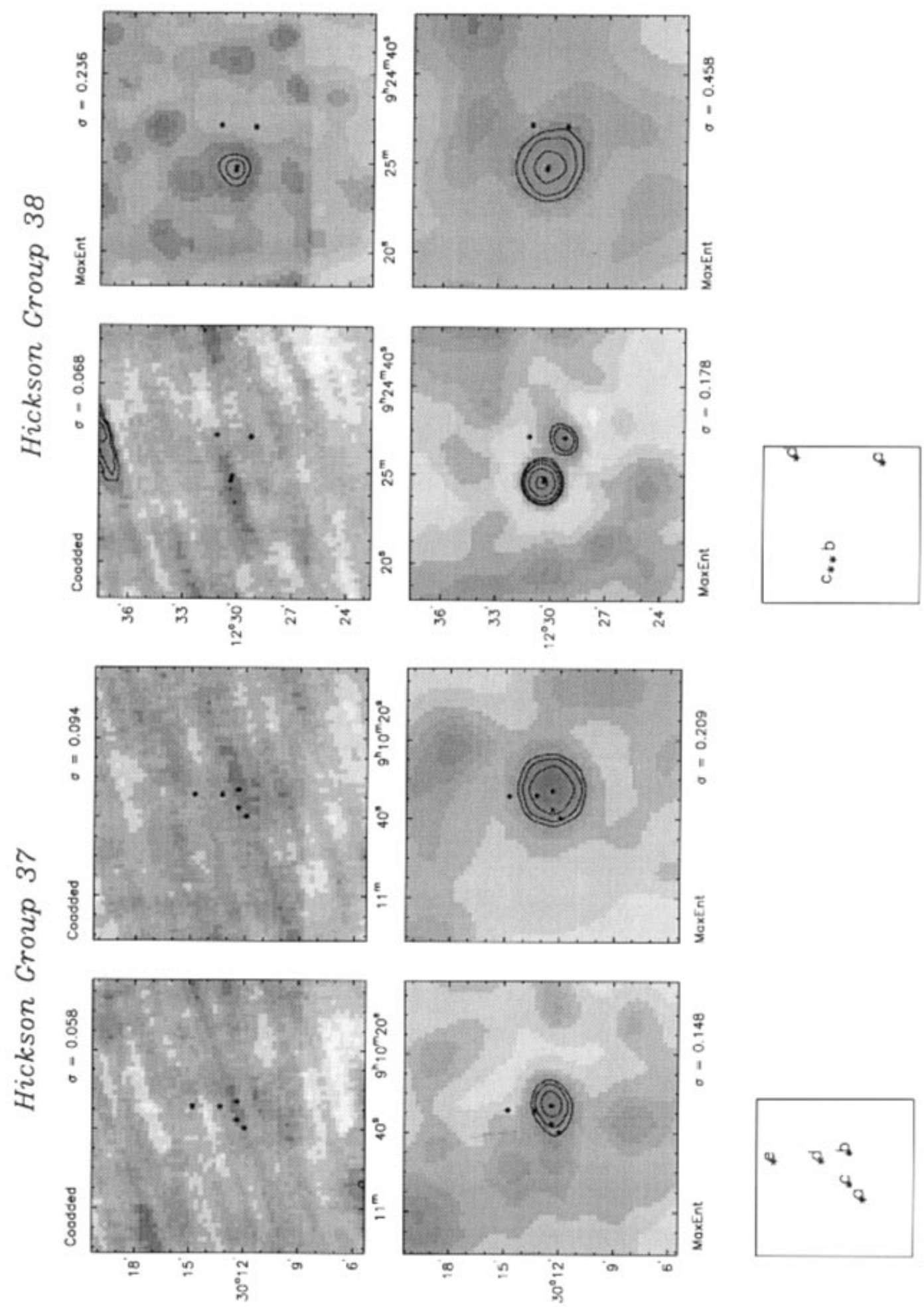

(To be seen in landscape) 

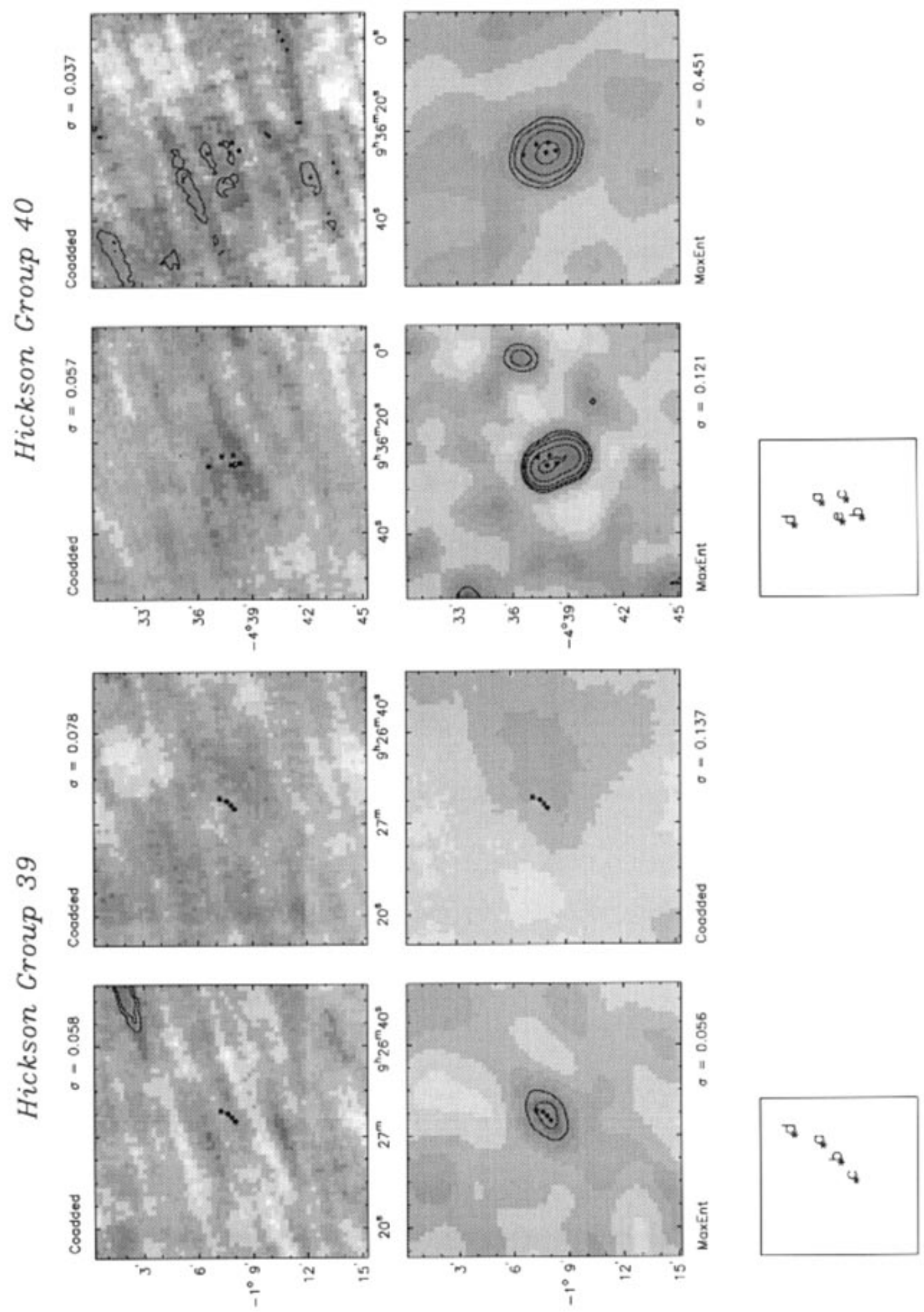

(To be seen in landscape) 

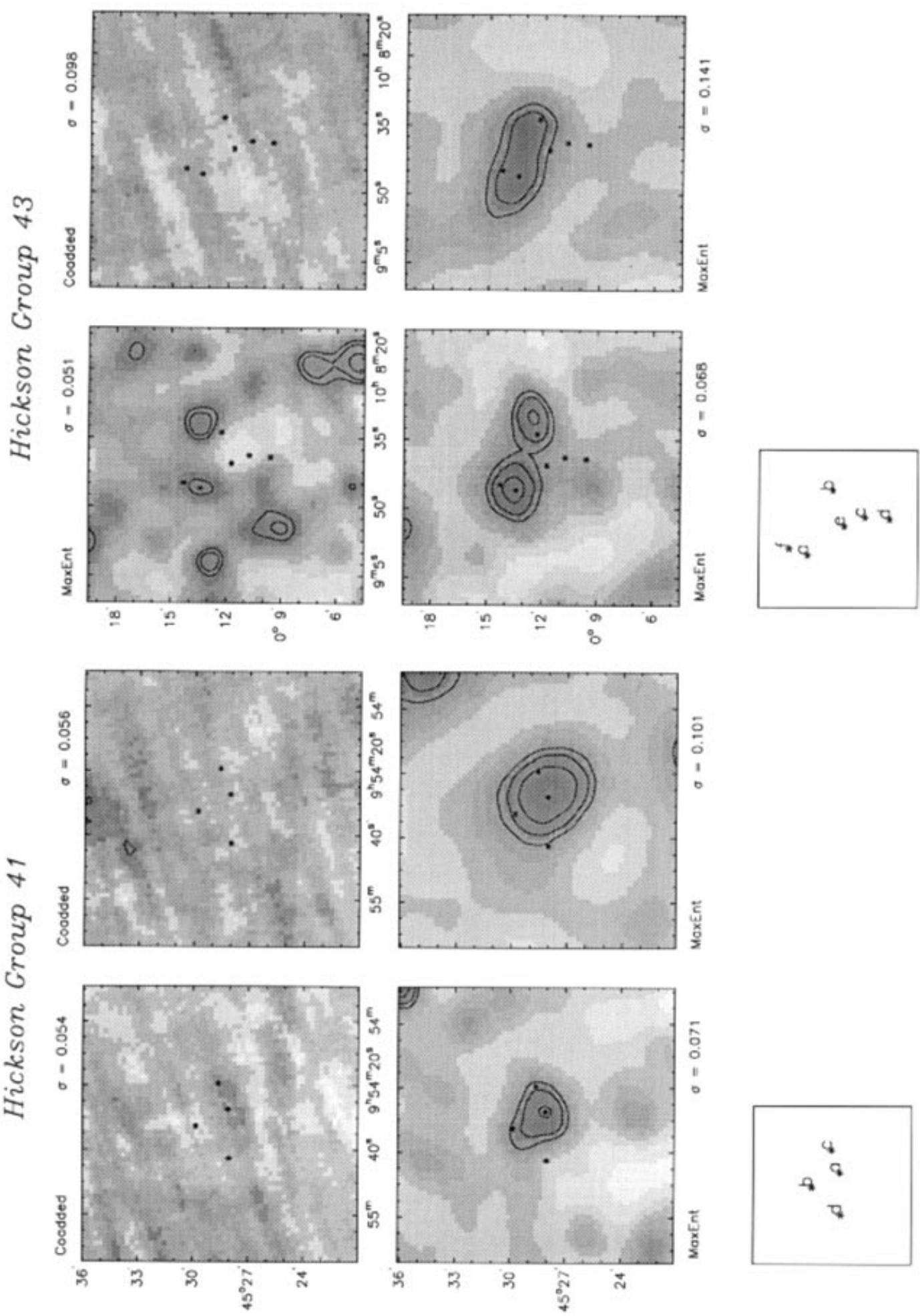

(To be seen in landscape) 

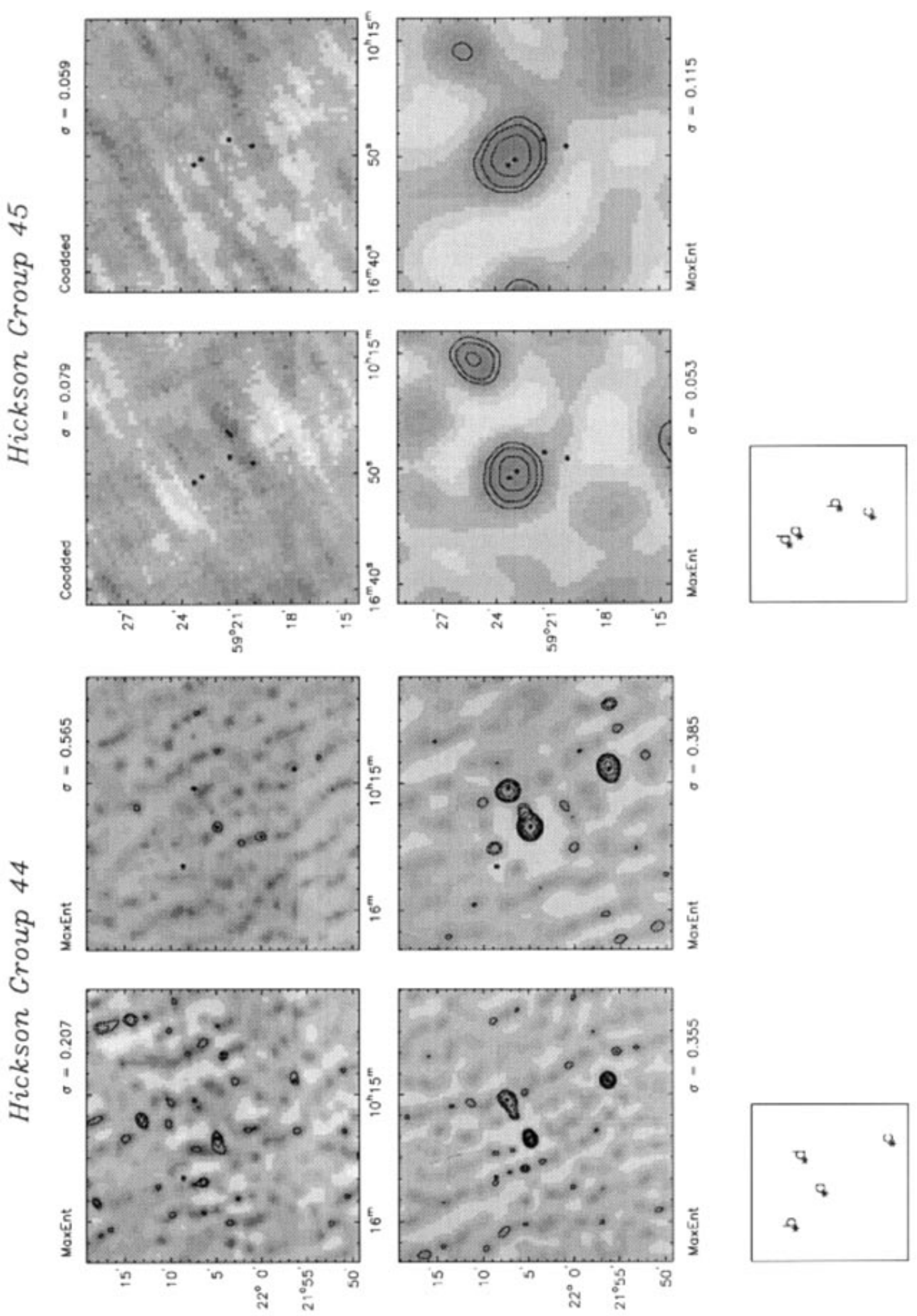

(To be seen in landscape) 

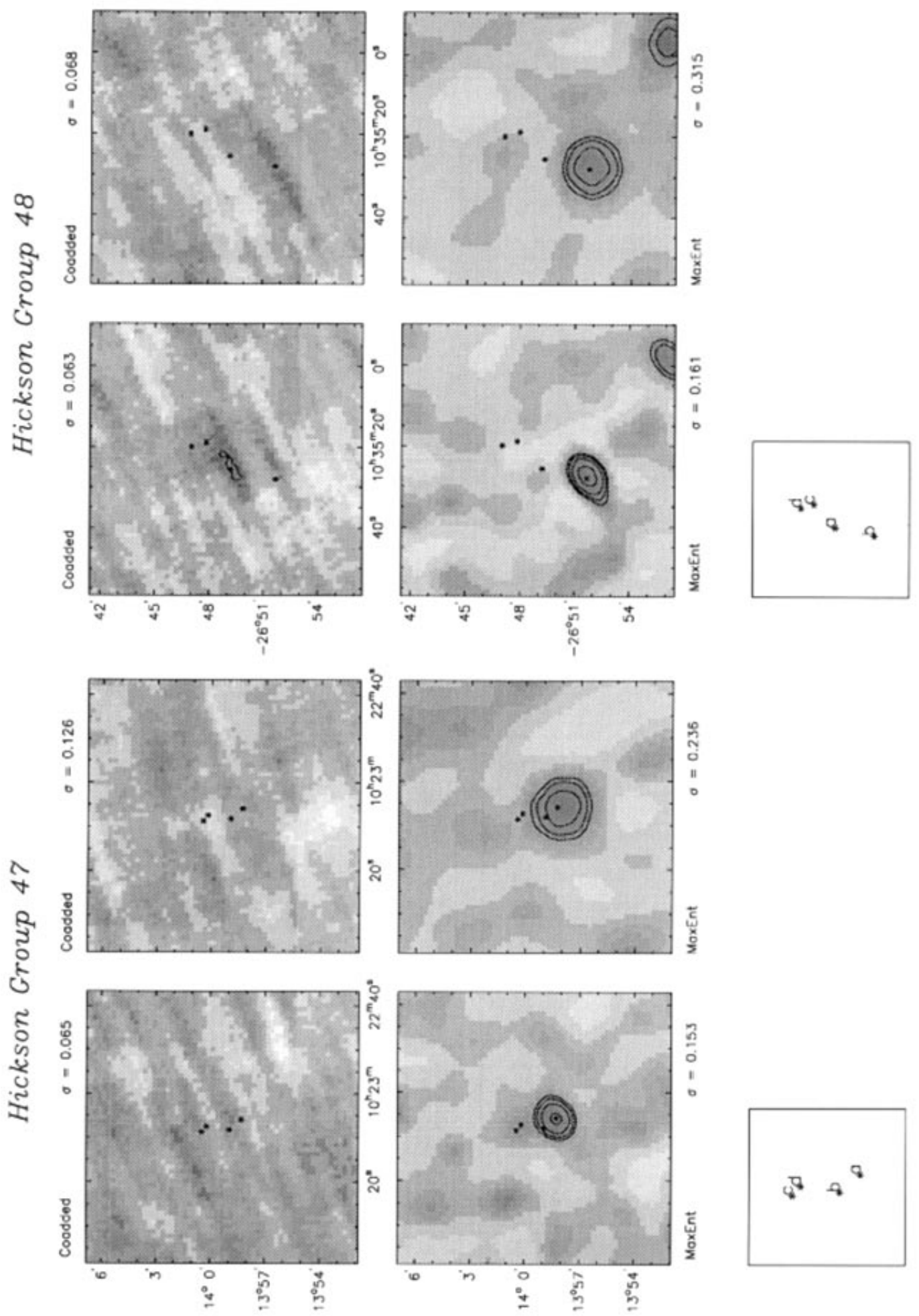

(To be seen in landscape) 

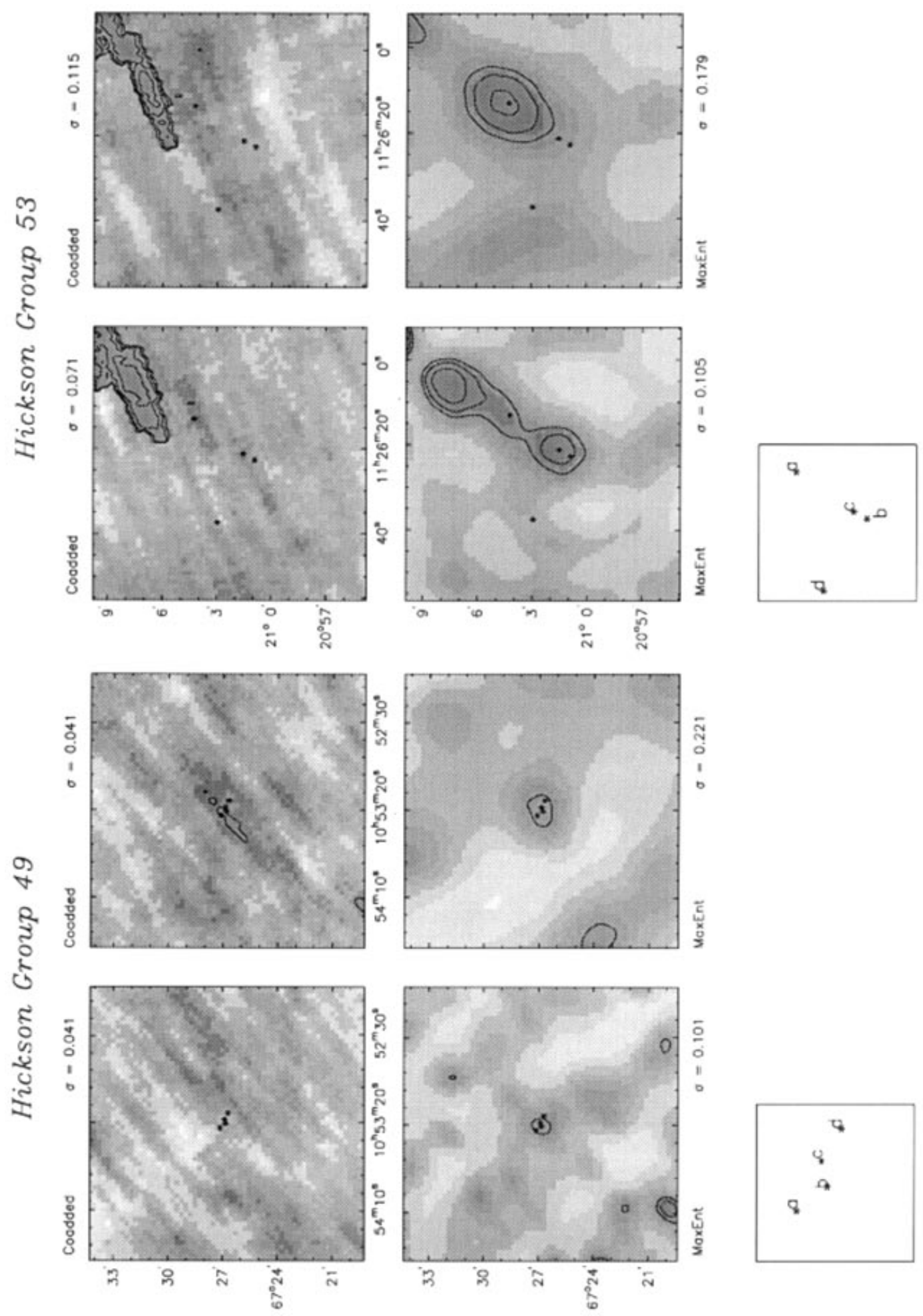

(To be seen in landscape) 

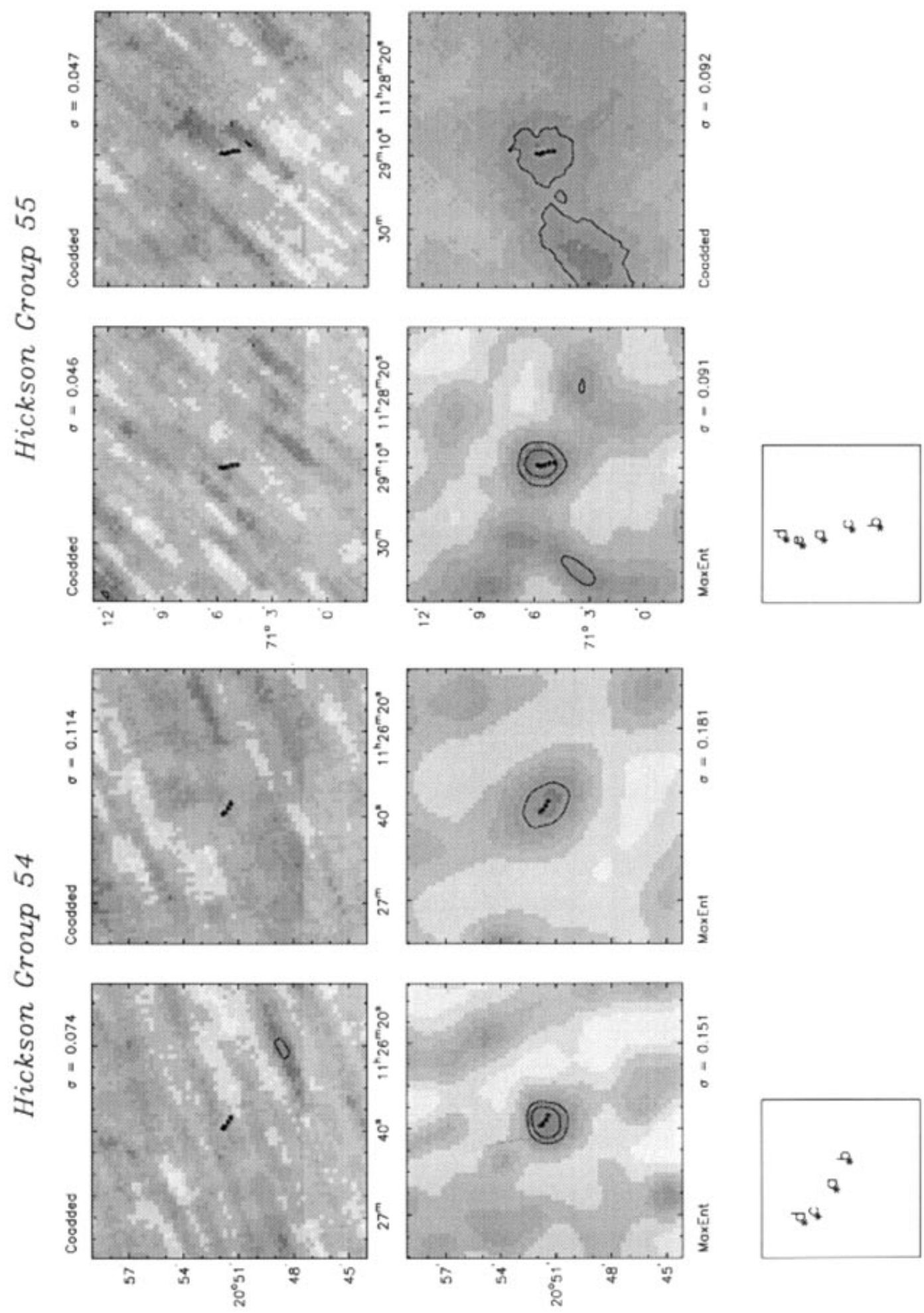

(To be seen in landscape) 

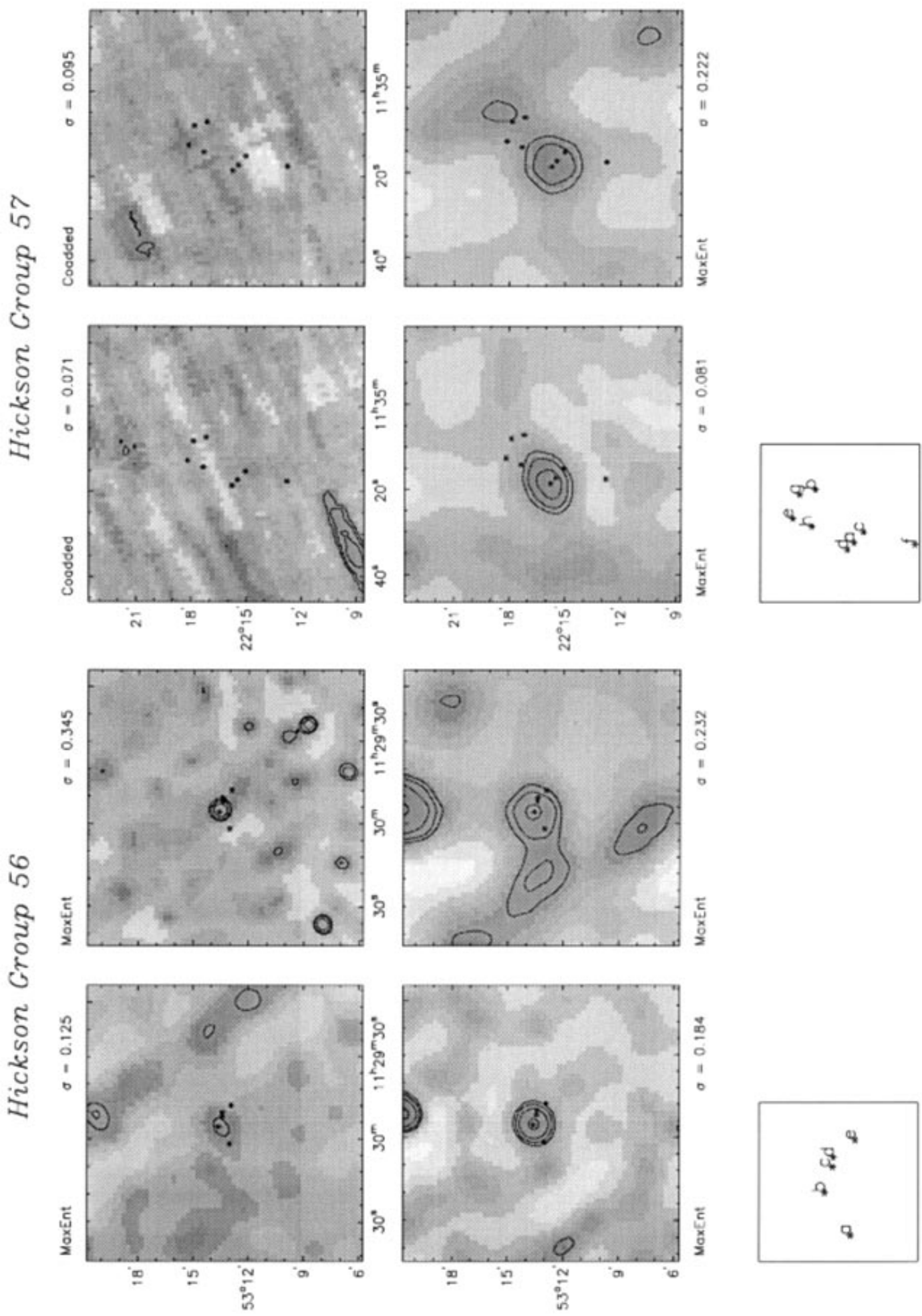

(To be seen in landscape) 

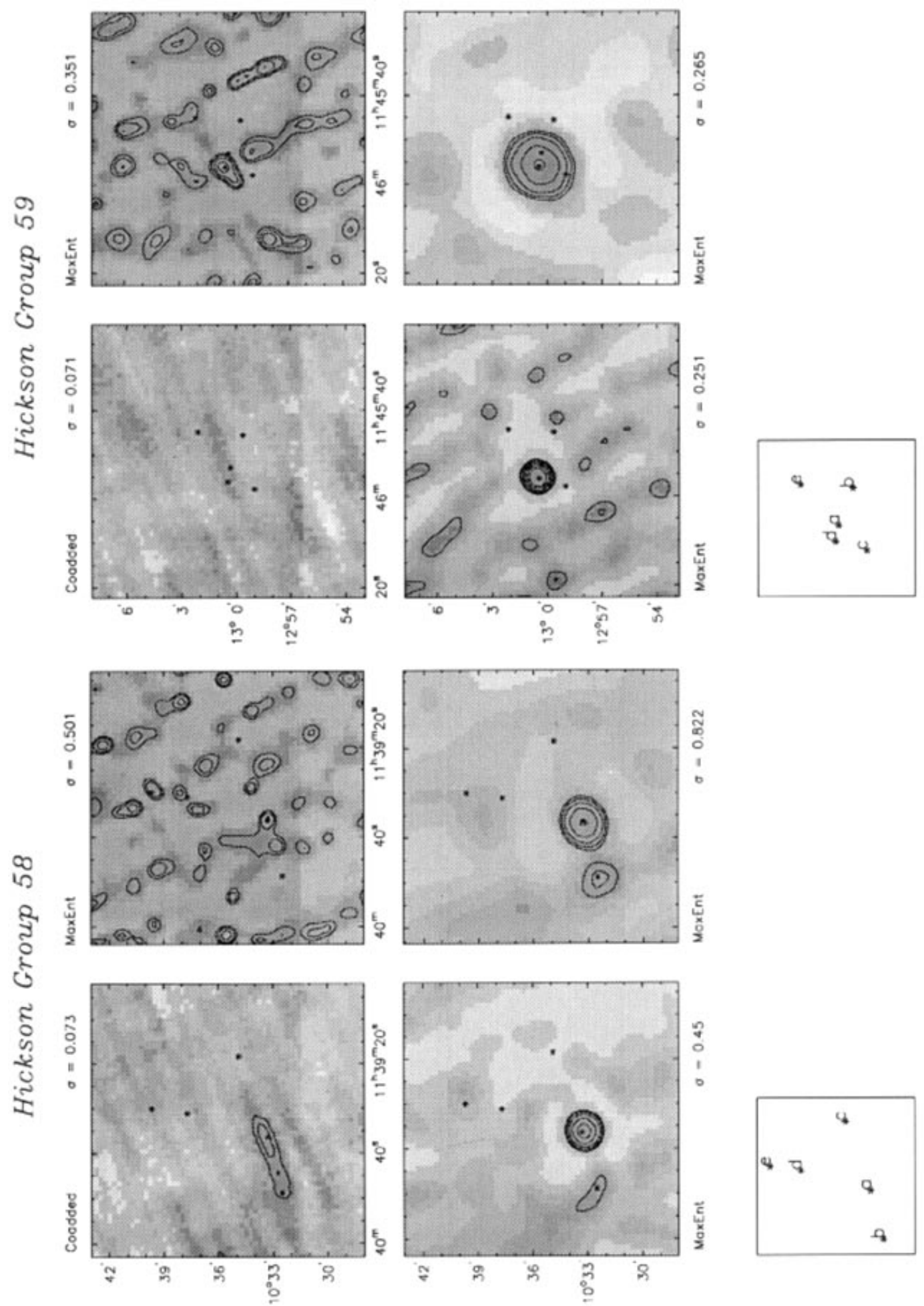

(To be seen in landscape) 

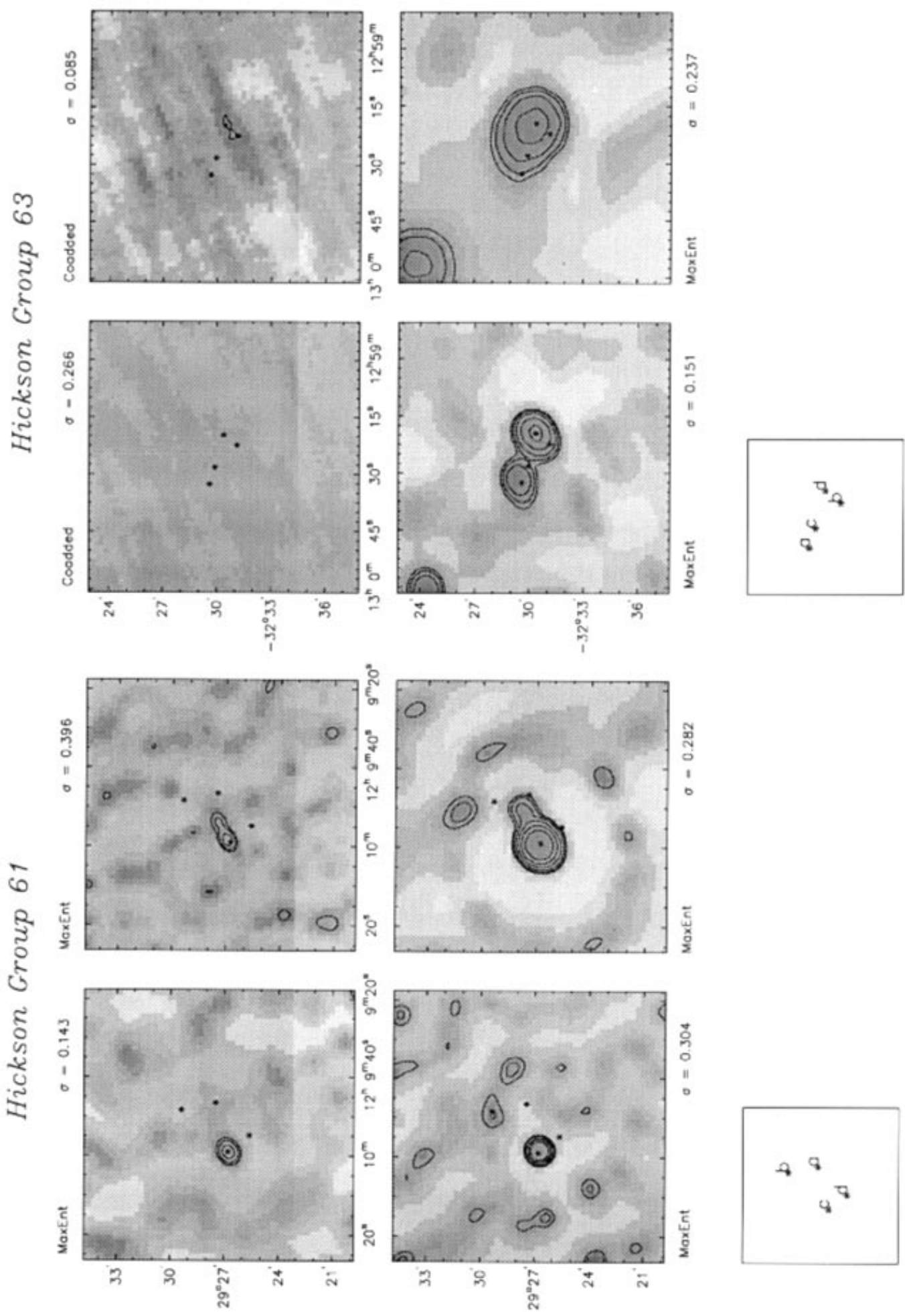

(To be seen in landscape) 

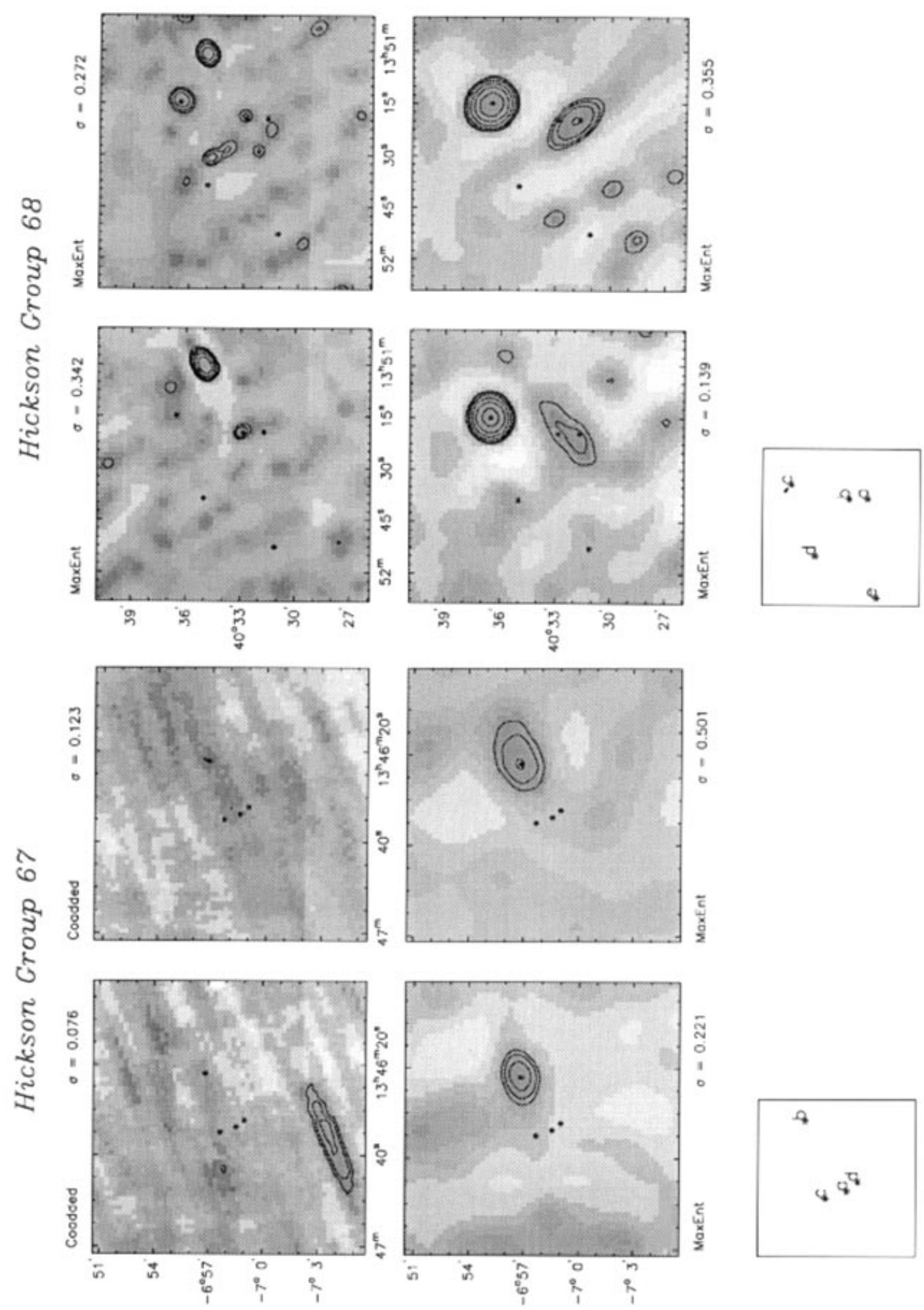

(To be seen in landscape) 

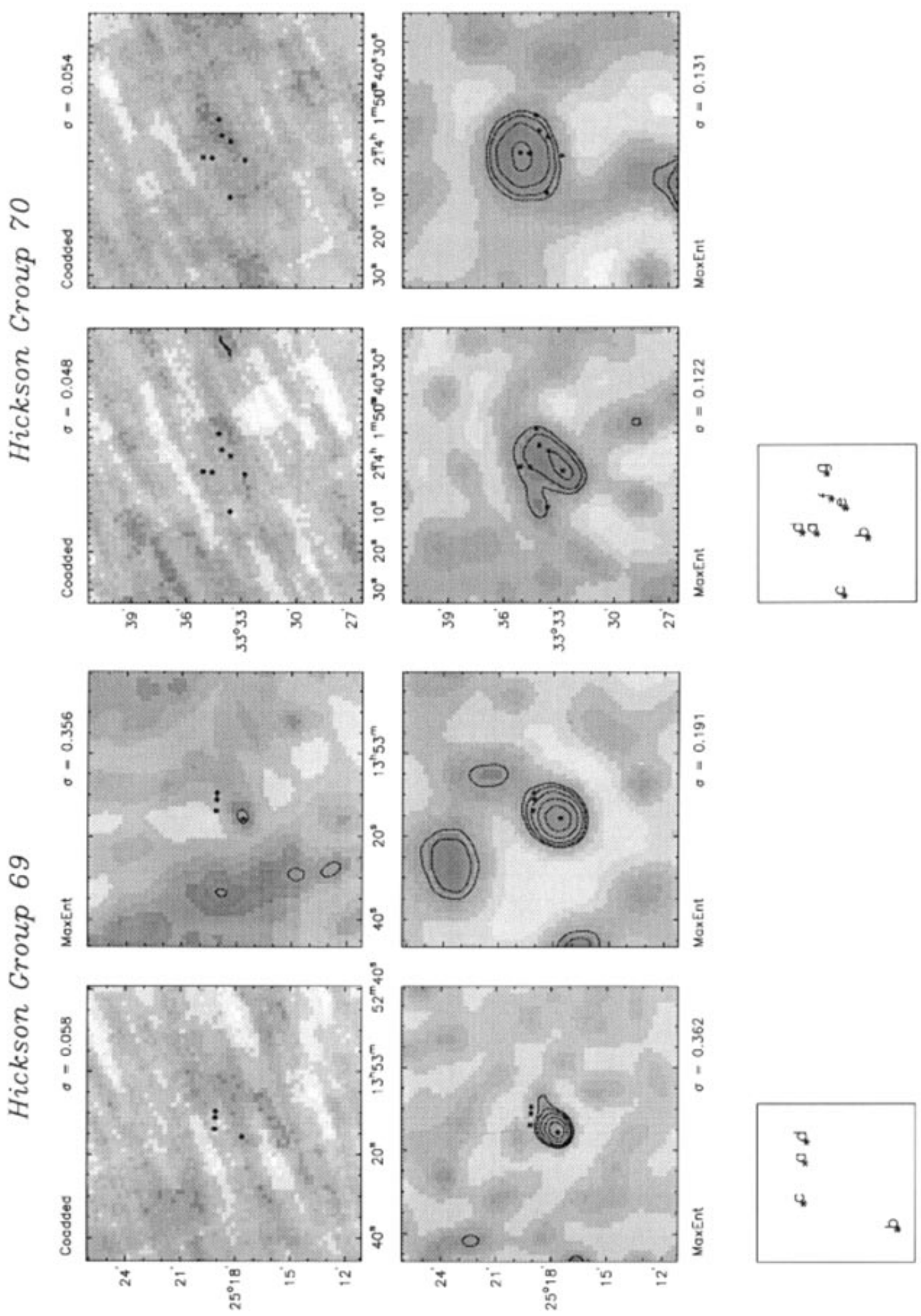

(To be seen in landscape) 

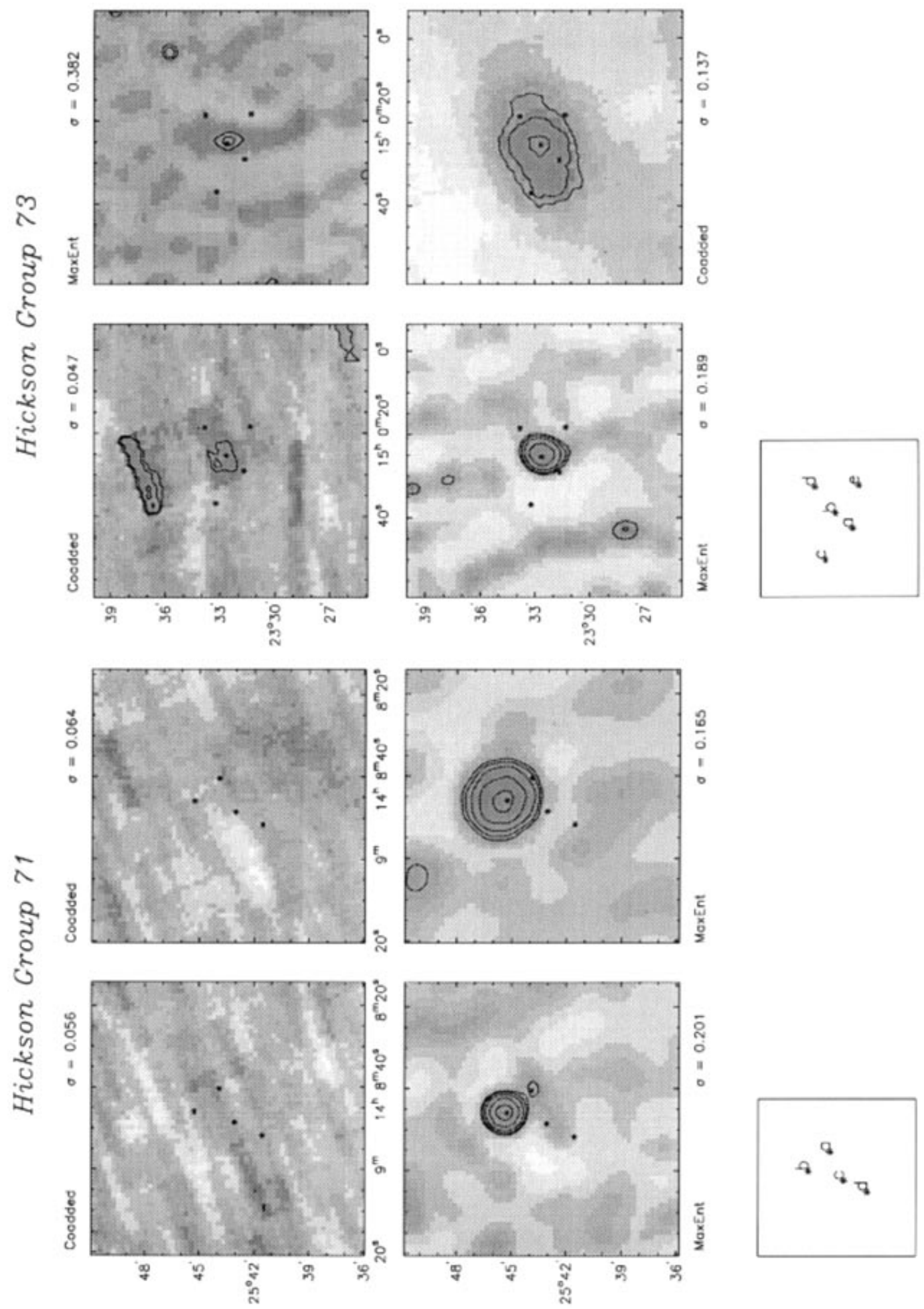

(To be seen in landscape) 

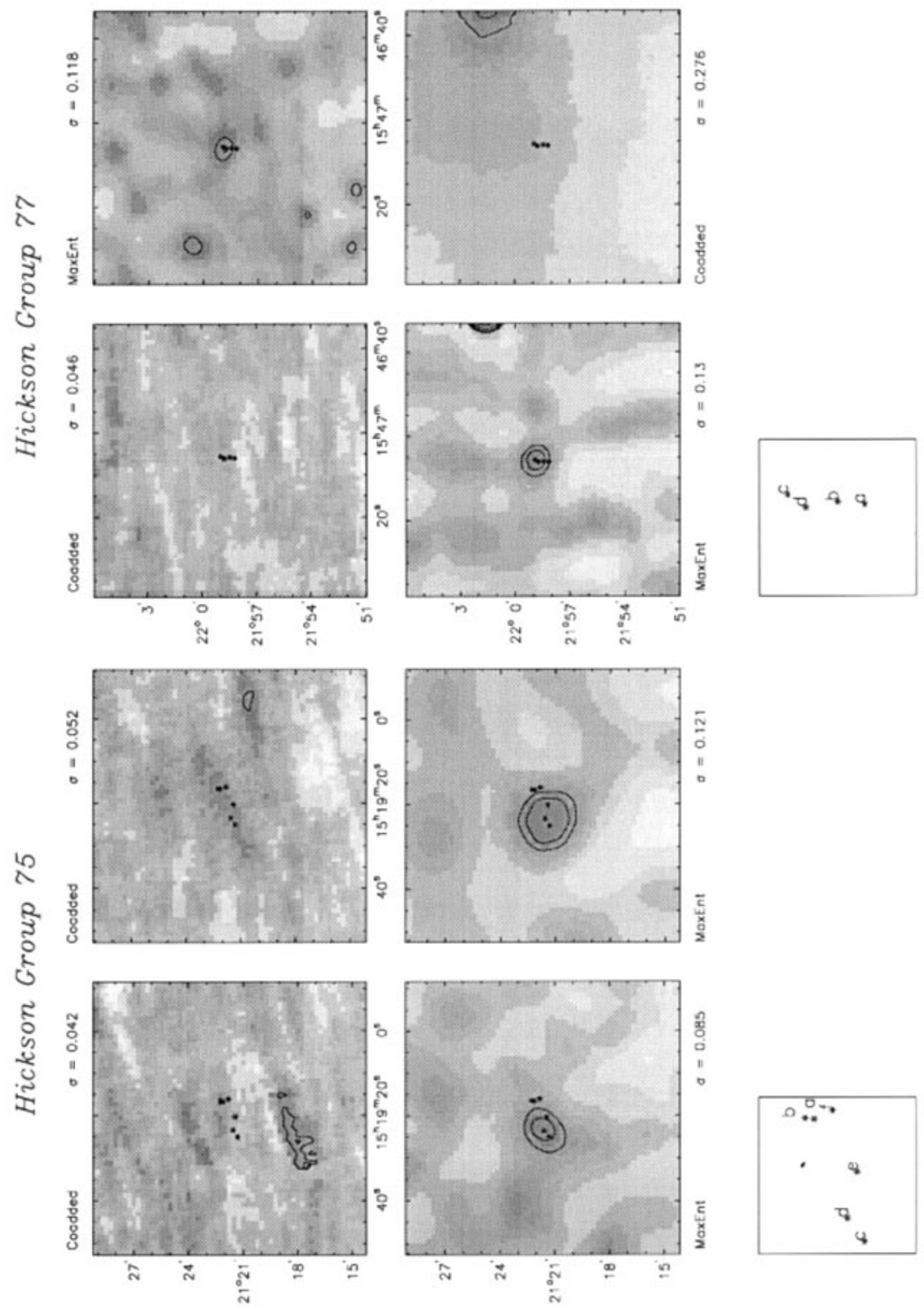

(To be seen in landscape) 


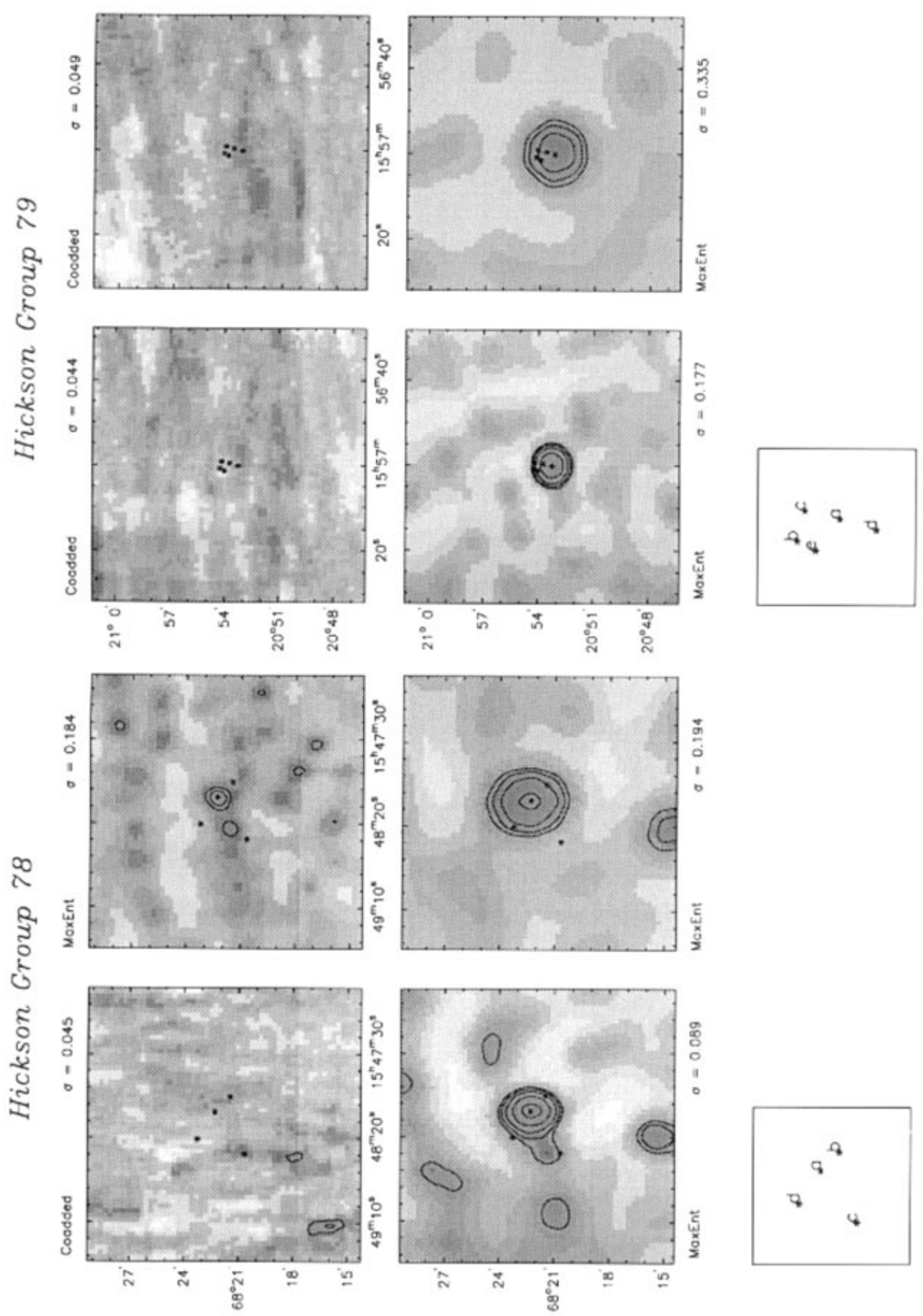

(To be seen in landscape) 

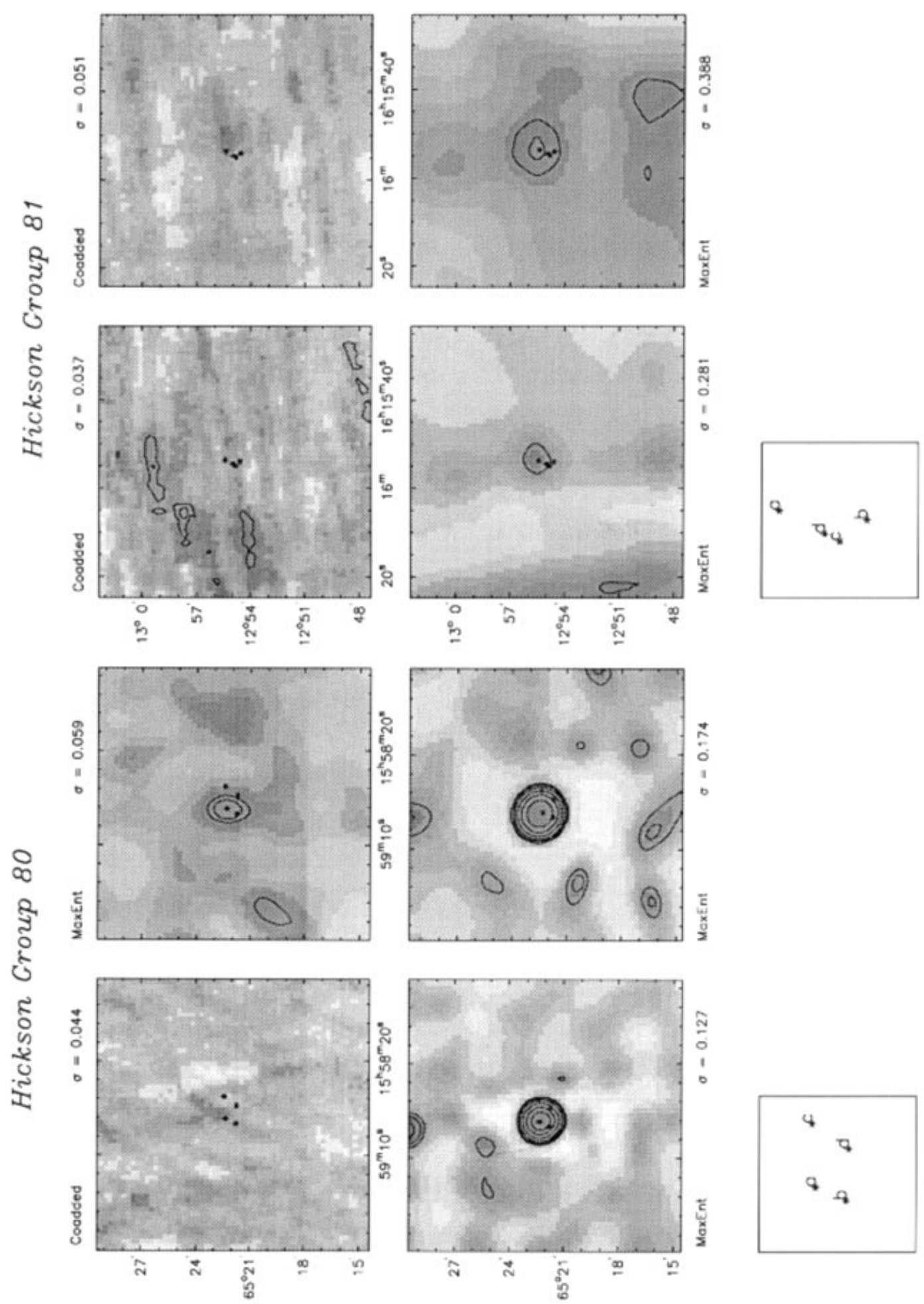

(To be seen in landscape) 

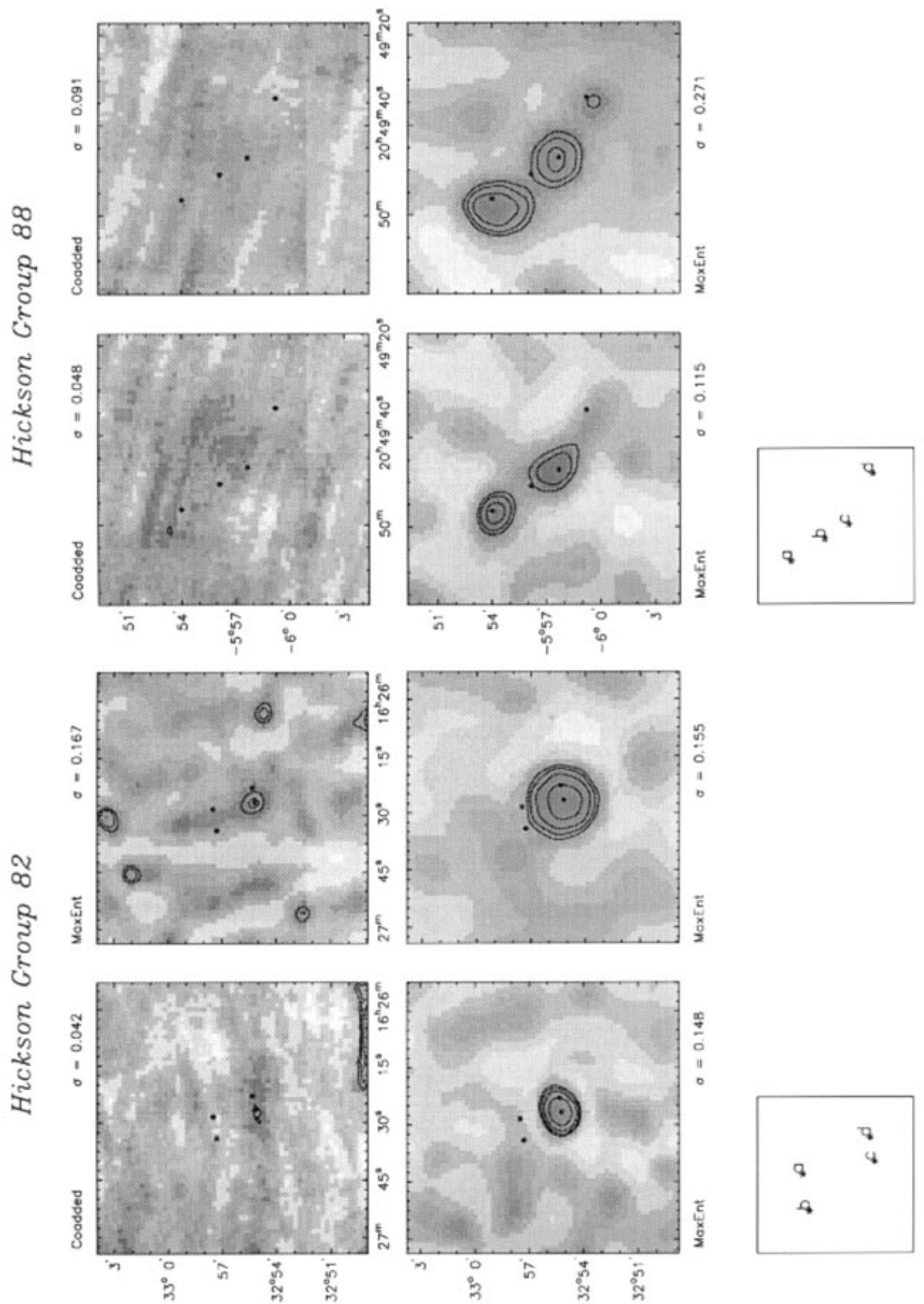

(To be seen in landscape) 

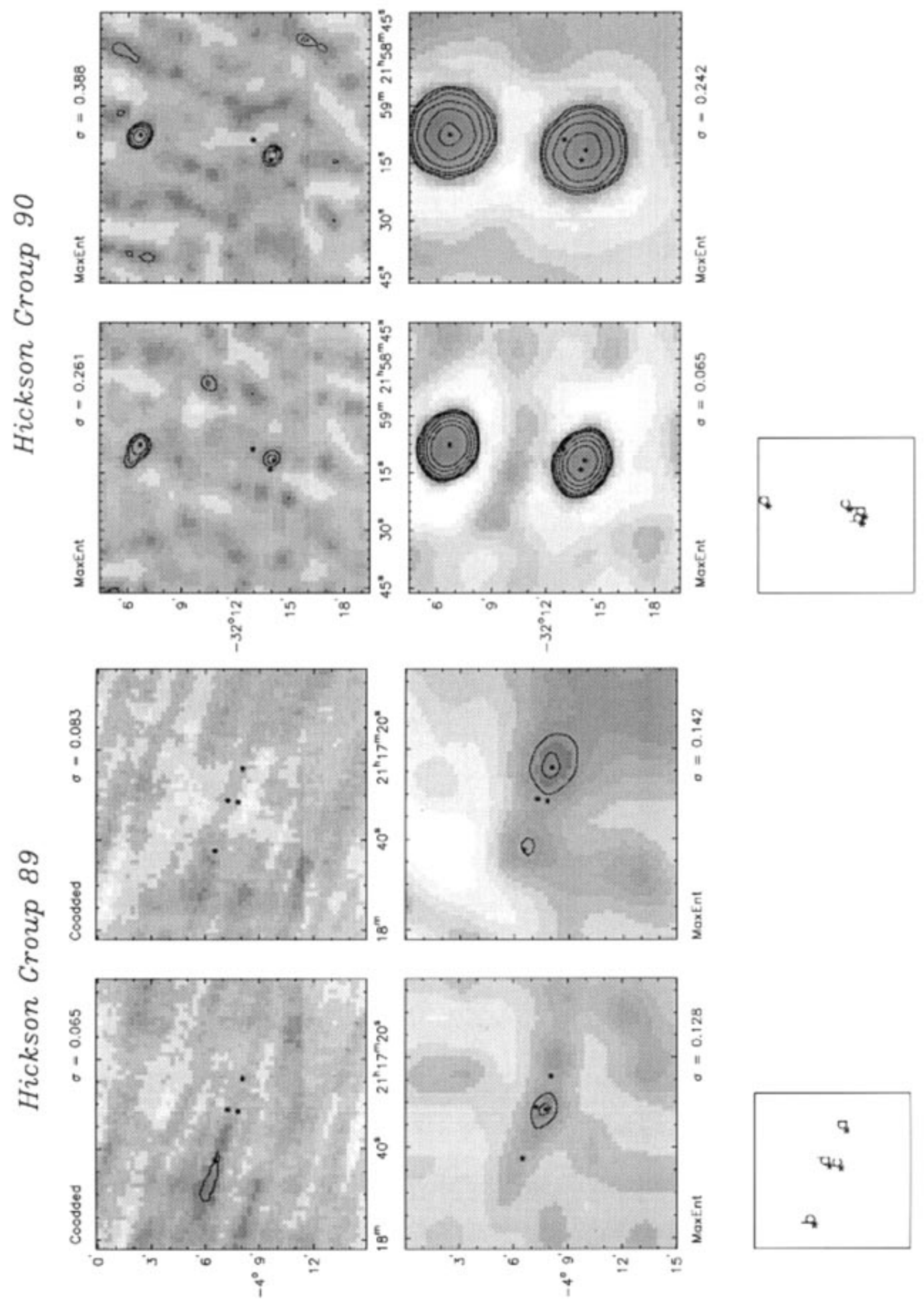

(To be seen in landscape) 

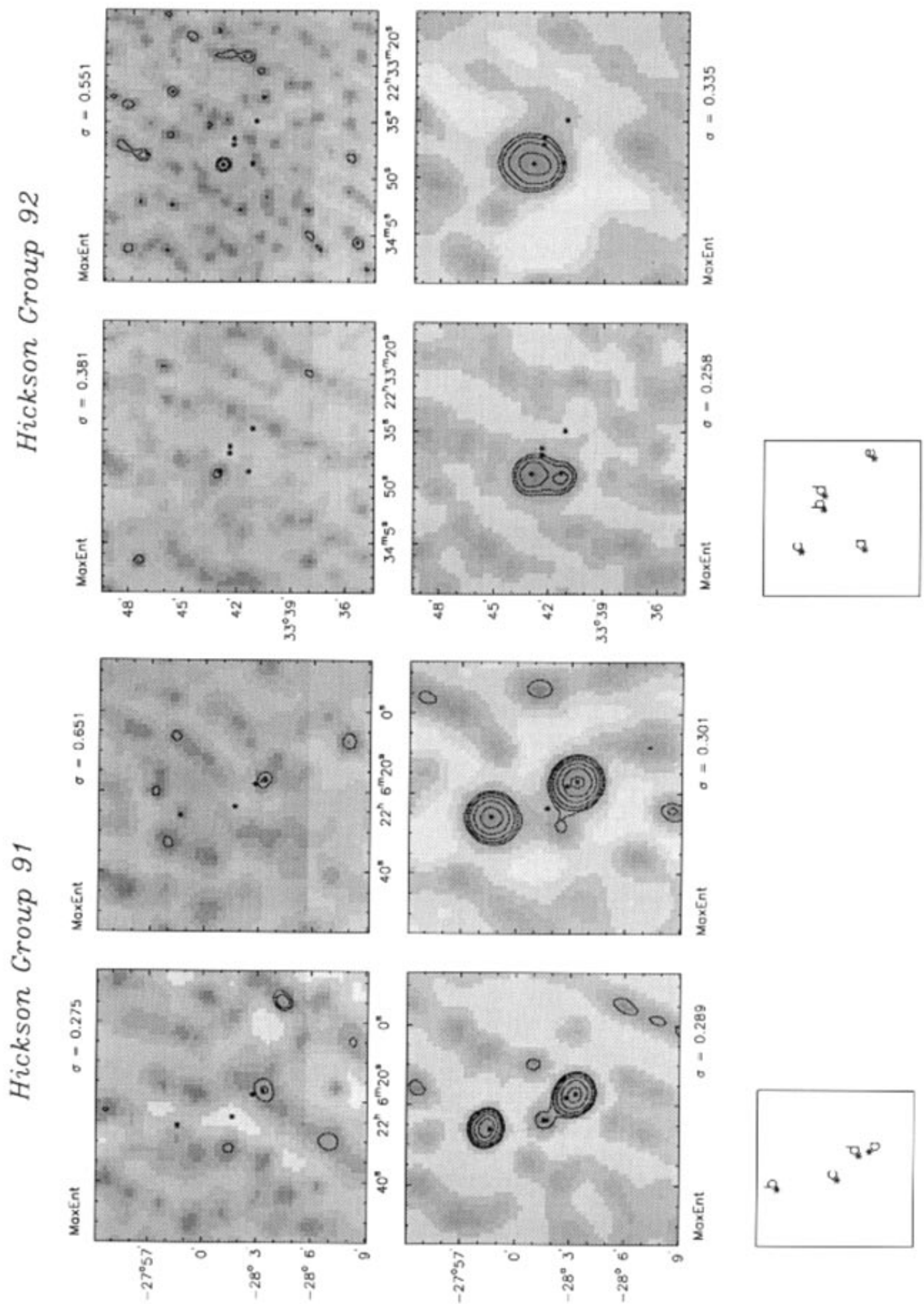

(To be seen in landscape) 

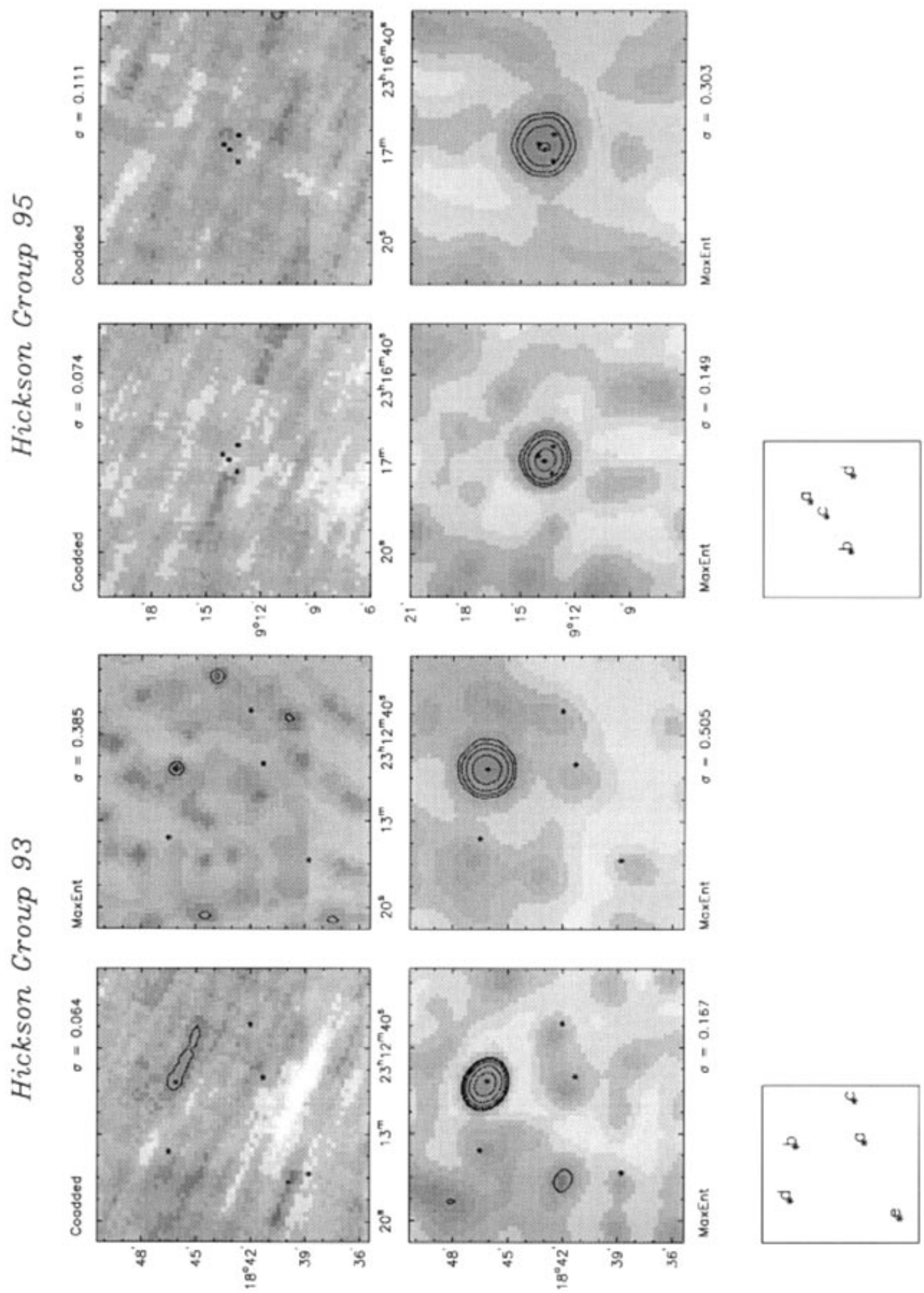

(To be seen in landscape) 

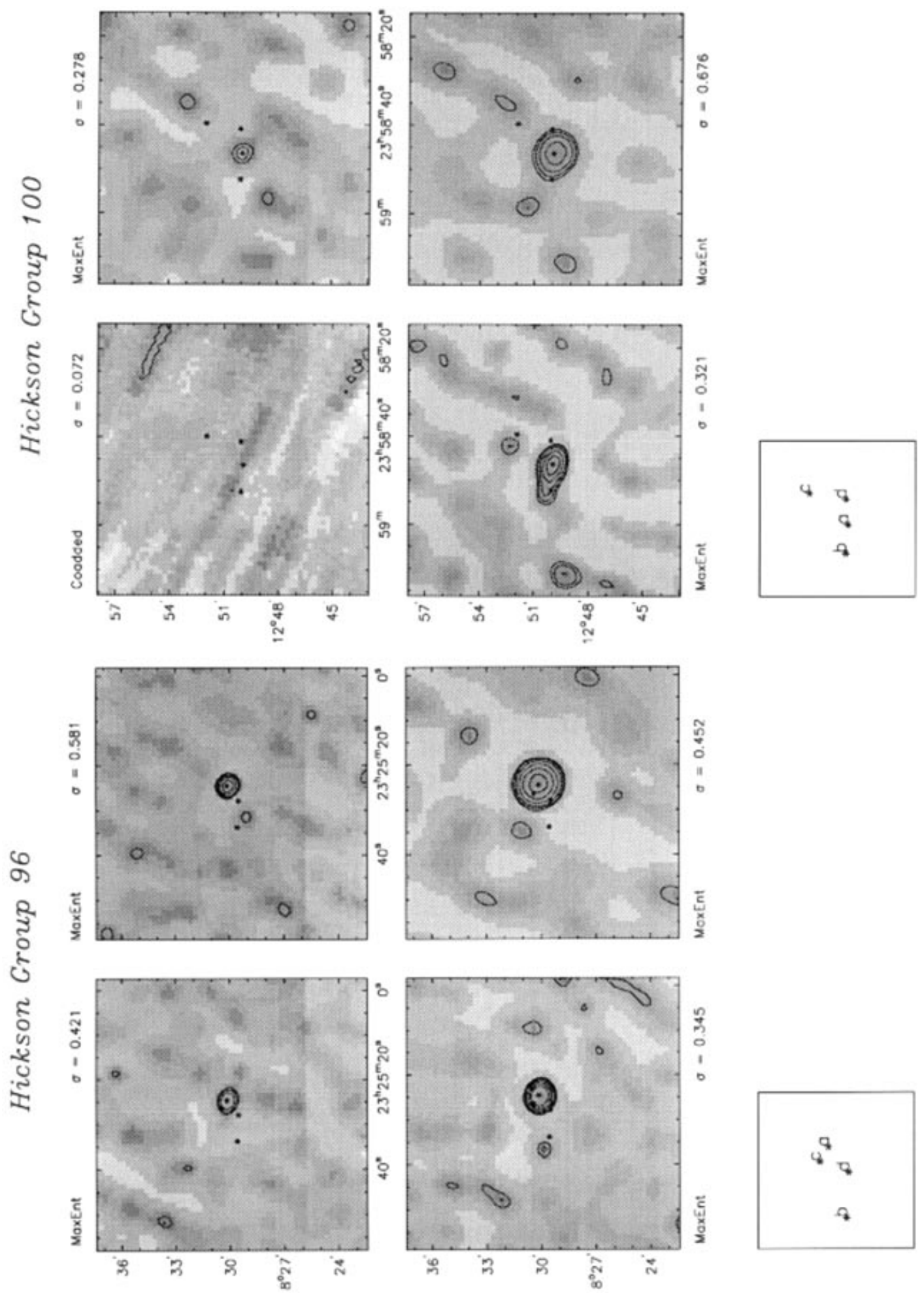

(To be seen in landscape) 